\title{
Weak Solutions and Their Kinetic Energy Regarding Time-Periodic Navier-Stokes Equations in Three Dimensional Whole-Space
}

\author{
Mads Kyed 1,2 (D) \\ 1 Fachbereich Maschinenbau, Flensburg University of Applied Sciences, Kanzleistrasse 91-93, \\ 24943 Flensburg, Germany; mads.kyed@hs-flensburg.de \\ 2 Faculty of Science and Engineering, Waseda University, 3-4-1 Ohkubo, Shinjuku-ku, Tokyo 169-8555, Japan
}

\begin{abstract}
The existence of weak time-periodic solutions to Navier-Stokes equations in three dimensional whole-space with time-periodic forcing terms are established. The solutions are constructed in such a way that the structural properties of their kinetic energy are obtained. No restrictions on either the size or structure of the external force are required.
\end{abstract}

Keywords: Navier-Stokes; time-periodic solutions

MSC: 35Q30; 76D05; 35B10

Citation: Kyed, M. Weak Solutions and Their Kinetic Energy Regarding Time-Periodic Navier-Stokes Equations in Three Dimensional Whole-Space. Mathematics 2021, 9 , 1528. https://doi.org/10.3390/ math9131528

Academic Editor: Christopher Goodrich

Received: 11 May 2021

Accepted: 18 June 2021

Published: 29 June 2021

Publisher's Note: MDPI stays neutral with regard to jurisdictional claims in published maps and institutional affiliations.

\section{Introduction}

We investigate weak solutions to the $\mathcal{T}$-time-periodic incompressible Navier-Stokes system in the three-dimensional whole-space

$$
\begin{cases}\partial_{t} u-\Delta u-\lambda \partial_{1} u+\nabla \mathfrak{p}+u \cdot \nabla u=f & \text { in } \mathbb{R} \times \mathbb{R}^{3}, \\ \operatorname{div} u=0 & \text { in } \mathbb{R} \times \mathbb{R}^{3}, \\ \lim _{|x| \rightarrow \infty} u(t, x)=0 & \\ u(t+\mathcal{T}, \cdot)=u(t, \cdot) & \end{cases}
$$

with a $\mathcal{T}$-time-periodic forcing term $f: \mathbb{R} \times \mathbb{R}^{3} \rightarrow \mathbb{R}^{3}, f(t+\mathcal{T}, \cdot)=f(t, \cdot)$. Here, $\mathbb{R}$ denotes the time axis. The unknowns $u: \mathbb{R} \times \mathbb{R}^{3} \rightarrow \mathbb{R}^{3}$ and $\mathfrak{p}: \mathbb{R} \times \mathbb{R}^{3} \rightarrow \mathbb{R}$ represent the Eulerian velocity field and pressure term, respectively, of a Navier-Stokes liquid. The system (1) is written in a frame of reference with velocity $\lambda \mathrm{e}_{1}, \lambda \in \mathbb{R}$, in order to model both the case of a fluid at rest at spatial infinity $(\lambda=0)$ and the case of a fluid with constant velocity at spatial infinity $(\lambda \neq 0)$.

To put the time-periodic Navier-Stokes problem (1) into context, it is worth comparing it to the corresponding initial-value problem obtained by replacing the time axis with an interval $(0, \mathcal{T})$ and the periodicity condition in (1) with an initial value $u(0, \cdot)=u_{0}$. Famously $[1,2]$, existence of a weak solution $u$ to the initial-value problem can be obtained in the Leray-Hopf class

$$
\mathrm{L}^{2}\left(0, \mathcal{T} ; \mathrm{D}^{1,2}\left(\mathbb{R}^{3}\right)\right) \cap \mathrm{L}^{\infty}\left(0, \mathcal{T} ; \mathrm{L}^{2}\left(\mathbb{R}^{3}\right)\right)
$$

for a suitable class of initial values and forcing terms. Here, $D^{1,2}\left(\mathbb{R}^{3}\right)$ denotes the homogeneous Sobolev space of locally integrable functions $u$ with $\nabla u \in \mathrm{L}^{2}\left(\mathbb{R}^{3}\right)$. Specifically, if the kinetic energy of the initial value is finite, existence of a weak solution in the class (2) can be obtained, that is, a solution whose kinetic energy given by the norm $\|u(t, \cdot)\|_{2}$ remains finite for all times $t$, which is, of course, what one expects from a physical point of view. 
The situation is different when we consider a solution to the time-periodic problem (1). This is perhaps best illustrated by considering a time-independent, and therefore trivially time-periodic, force $f$. In this case, existence of a corresponding steady-state solution $u$, which is also trivially time-periodic, is well-known. It is also well-known that steady-state solutions to the Navier-Stokes problem in unbounded domains $\Omega$ in general do not belong to $\mathrm{L}^{2}(\Omega)$. In fact, the information available on the structure of steady-state solutions to the Navier-Stokes system in unbounded domains is very comprehensive (at least in the case $\lambda \neq 0$ ); see for example [3] (Chapter X). Roughly speaking, the unbounded domain allows for velocity distributions of steady-state solutions with infinite kinetic energy. Since steady-states are special cases of time-periodic solutions, we conclude that solutions to (1), in general, do not belong to the Leray-Hopf class (2). The question therefore arises as to which function space is best suited to study weak solutions to (1) with energy methods. The main goal of this article is to give an answer to this question.

We introduce the simple time-average projection

$$
\mathcal{P} u(x):=\frac{1}{\mathcal{T}} \int_{0}^{\mathcal{T}} u(t, x) \mathrm{d} t, \quad \mathcal{P}_{\perp} u(t, x):=u(t, x)-\mathcal{P} u(x)
$$

and use it to decompose a solution $u=\mathcal{P} u+\mathcal{P}_{\perp} u$ to (1) into a steady-state part $\mathcal{P} u$, independent on time, and a so-called purely oscillatory part $\mathcal{P}_{\perp} u$. A Galerkin method is employed to establish the existence of a weak time-periodic solution $u$ to (1) with a steadystate part $\mathcal{P} u \in \mathrm{D}^{1,2}\left(\mathbb{R}^{3}\right) \cap \mathrm{L}^{6}\left(\mathbb{R}^{3}\right)$ in the function space intrinsic to steady-state solutions of the Navier-Stokes problem, and a purely oscillatory part $\mathcal{P}_{\perp} u$ in the Leray-Hopf class (2) with finite kinetic energy at all times. In other words, we retain the Leray-Hopf class as a canonical energy space for the purely oscillatory part of the solution. More precisely, the following theorem is established as the main theorem:

Theorem 1. Let $f: \mathbb{R} \times \mathbb{R}^{3} \rightarrow \mathbb{R}^{3}$ be $\mathcal{T}$-time-periodic with $f \in \mathrm{L}^{2}\left(0, \mathcal{T} ; \mathrm{D}_{0}^{-1,2}\left(\mathbb{R}^{3}\right)\right)$ and $\lambda \in \mathbb{R}$. There exists a weak $\mathcal{T}$-time-periodic solution $u: \mathbb{R} \times \mathbb{R}^{3} \rightarrow \mathbb{R}^{3}$ to (1) that satisfies

$$
\begin{aligned}
& \mathcal{P} u \in \mathrm{D}^{1,2}\left(\mathbb{R}^{3}\right) \cap \mathrm{L}^{6}\left(\mathbb{R}^{3}\right), \\
& \mathcal{P}_{\perp} u \in \mathrm{L}^{2}\left(0, \mathcal{T} ; \mathrm{D}^{1,2}\left(\mathbb{R}^{3}\right)\right) \cap \mathrm{L}^{\infty}\left(0, \mathcal{T} ; \mathrm{L}^{2}\left(\mathbb{R}^{3}\right)\right) .
\end{aligned}
$$

Here, a weak solution to (1) is defined as a $\mathcal{T}$-time-periodic vector field $u: \mathbb{R} \times \mathbb{R}^{3} \rightarrow \mathbb{R}^{3}$ with $u \in \mathrm{L}^{2}\left(0, \mathcal{T} ; \mathrm{D}^{1,2}\left(\mathbb{R}^{3}\right)\right)$ and $\operatorname{div} u=0$ satisfying

$$
\int_{0}^{\mathcal{T}} \int_{\mathbb{R}^{3}}-u \cdot \partial_{t} \Phi+\nabla u: \nabla \Phi-\lambda \partial_{1} u \cdot \Phi+(u \cdot \nabla u) \cdot \Phi \mathrm{d} x \mathrm{~d} t=\int_{0}^{\mathcal{T}}\langle f, \Phi\rangle \mathrm{d} t
$$

for all $\mathcal{T}$-time-periodic test functions $\Phi \in C^{\infty}\left(\mathbb{R} \times \mathbb{R}^{3}\right)^{3}$ with $\Phi \in C_{0}^{\infty}\left([0, \mathcal{T}] \times \mathbb{R}^{3}\right)$. Moreover, $A: B$ denotes the standard inner product of square matrices $A$ and $B$.

Existence of a weak solution is obtained without any restrictions on the size of the data $f$. Utilizing a Fourier expansion in time, we shall employ a Galerkin approach based on approximating subspaces that are finite-dimensional both in time and space. The time discretization allows us to single out the steady-state part $\mathcal{P} u$ as the zeroth order Fourier mode at any level of the investigation and analyze it separately. In contrast to the corresponding initial-value problem, the absence of a prescribed initial-value makes the task of showing $\mathcal{P}_{\perp} u \in \mathrm{L}^{\infty}\left(0, \mathcal{T} ; \mathrm{L}^{2}\left(\mathbb{R}^{3}\right)\right)$ in the time-periodic case more delicate than one would perhaps expect from the relatively simple argument used to establish $u \in \mathrm{L}^{\infty}\left(0, \mathcal{T} ; \mathrm{L}^{2}\left(\mathbb{R}^{3}\right)\right)$ for the initial-value problem. We briefly elaborate on this matter in Remark 5.

There are two important implications of the property $\mathcal{P} \perp u \in \mathrm{L}^{\infty}\left(0, \mathcal{T} ; \mathrm{L}^{2}\left(\mathbb{R}^{3}\right)\right)$. In physical terms, the property implies that the weak time-periodic solution $u$ can be written as a sum of a steady-state part $\mathcal{P} u$, and a time-dependent part $\mathcal{P} \perp u$ with finite kinetic 
energy. In mathematical terms, the property is important for the development of further regularity properties. Experience from the initial-value problem, which has famously been studied intensively over the years, shows that additional regularity for $u$ in the time variable is the biggest obstacle. Since $u=\mathcal{P} u+\mathcal{P}_{\perp} u$ and $\mathcal{P} u$ is time-independent, $\mathcal{P}_{\perp} u \in \mathrm{L}^{\infty}\left(0, \mathcal{T} ; \mathrm{L}^{2}\left(\mathbb{R}^{3}\right)\right)$ is as good a starting point for an investigation into further time regularity as $u \in \mathrm{L}^{\infty}\left(0, \mathcal{T} ; \mathrm{L}^{2}\left(\mathbb{R}^{3}\right)\right)$ is for the initial-value problem. In other words, Theorem 1 "levels the playing field" for the time-periodic and initial-value Navier-Stokes problem in this respect. The famous problem of establishing higher order regularity for weak solutions to the initial-value Navier-Stokes problem remains unsolved to this date, however, and it appears that the same problem for the time-periodic problem is just as challenging.

The first rigorous investigation of the time-periodic Navier-Stokes equations in the three-dimensional whole-space was carried out by Maremonti [4], who established the existence of a solution to (1) in the space $\mathrm{L}^{\infty}\left(0, T ; \mathrm{L}^{3}\left(\mathbb{R}^{3}\right)\right)$ under a structural condition $f=\operatorname{curl} \psi$ imposed on the data. Subsequently, existence results were established in other unbounded three-dimensional domains [5-9]. Theorem 1, which is based on ideas from [10], is the first result in which the existence of a weak solution was established in the natural energy space (3)-(4). A comprehensive overview of the time-periodic Navier-Stokes equations can be found in [11].

The proof of Theorem 1 is presented in Section 3. In Section 4, further regularity properties of weak solutions are established. Specifically, we show that the purely oscillatory part $\mathcal{P}_{\perp} u$ is continuous in time when considered as a mapping in time with values in the space $L^{2}\left(\mathbb{R}^{3}\right)$ endowed with the weak topology. Finally, in Section 5 , the existence of a pressure $\mathfrak{p}$ corresponding to a weak solution $u$ is established with the property that $(u, \mathfrak{p})$ is a distributional solution to (1). We thereby underline that the notion (5) of a weak $\mathcal{T}$-time-periodic solution is meaningful.

\section{Preliminaries}

\subsection{Notation}

Constants in the scope of a single proof are denoted by a lower case $c$, and constants in the scope of the whole article are denoted by an upper case $C$. The notation $c=$ $c\left(\lambda_{1}, \ldots, \lambda_{n}\right)$ is employed to denote dependency of the constant on parameters $\lambda_{1}, \ldots, \lambda_{n}$.

\subsection{Sobolev Spaces}

For any domain $\Omega \subset \mathbb{R}^{3}$ and $m \in \mathbb{N}_{0}$, we denote, by $\mathrm{W}^{m, q}(\Omega)$, the classical inhomogeneous Sobolev space and, by $\|\cdot\|_{m, q}$, its norm. By $\mathrm{D}^{m, q}(\Omega)$, we denote the homogeneous Sobolev space

$$
\mathrm{D}^{m, q}(\Omega):=\left\{u \in \mathrm{L}_{\mathrm{loc}}^{1}(\Omega) \mid \partial^{\alpha} u \in \mathrm{L}^{q}(\Omega) \text { for all } \alpha \in \mathbb{N}_{0}^{3} \text { with }|\alpha|=m\right\}
$$

equipped with the semi-norm

$$
|u|_{m, q}:=\left(\sum_{|\alpha|=m} \int_{\Omega}\left|\partial^{\alpha} u(x)\right|^{q} \mathrm{~d} x\right)^{\frac{1}{q}} .
$$

Clearly, $|\cdot|_{m, q}$ defines a norm on $C_{0}^{\infty}(\Omega)$, and we let

$$
\mathrm{D}_{0}^{m, q}(\Omega):=\left.{\overline{C_{0}^{\infty}(\Omega)}}^{|\cdot|}\right|_{m, q}
$$

denote the closure with respect to this norm. We use the notation $\mathrm{D}_{0}^{-m, q}(\Omega)$ to denote the dual space of $\mathrm{D}_{0}^{m, q^{\prime}}(\Omega)$, and $|\cdot|_{-m, q}$ to denote its norm. 
We use $C_{0, \sigma}^{\infty}(\Omega)$ to denote the space of solenoidal vector fields in $C_{0}^{\infty}(\Omega)^{3}$, that is,

$$
C_{0, \sigma}^{\infty}(\Omega):=\left\{u \in C_{0}^{\infty}(\Omega)^{3} \mid \operatorname{div} u=0\right\},
$$

and introduce the spaces

$$
\mathrm{L}_{\sigma}^{q}(\Omega):={\overline{C_{0, \sigma}^{\infty}(\Omega)}}^{\|\cdot\|_{q}}, \mathrm{D}_{0, \sigma}^{m, q}(\Omega):=\left.{\overline{C_{0, \sigma}^{\infty}(\Omega)}}^{|\cdot|}\right|_{m, q}, \mathrm{~W}_{0, \sigma}^{m, q}(\Omega):={\overline{C_{0, \sigma}^{\infty}(\Omega)}}^{\|\cdot\|_{m, q}}
$$

of solenoidal vector fields.

We recall an embedding theorem that is not always found in standard literature:

Lemma 1. Let $q \in[1,3), r \in[q, 3 q /(3-q)]$, and $\lambda:=\frac{3(r-q)}{r q}$.

Then

$$
\forall u \in C_{0}^{\infty}\left(\mathbb{R}^{3}\right): \quad\|u\|_{r} \leq C_{1}\|u\|_{q}^{1-\lambda}\|\nabla u\|_{q}^{\lambda}
$$

with $C_{1}=C_{1}(\lambda, q, r)$.

Proof. See for example [12] (Lemma II.2.2).

As a direct consequence of Lemma 1, it follows that

$$
\forall u \in \mathrm{D}_{0}^{1,2}\left(\mathbb{R}^{3}\right):\|u\|_{\mathrm{L}^{6}\left(\mathbb{R}^{3}\right)} \leq C_{1}\|\nabla u\|_{\mathrm{L}^{2}\left(\mathbb{R}^{3}\right)} .
$$

Consequently, we can identify $\mathrm{D}_{0}^{1,2}\left(\mathbb{R}^{3}\right)=\mathrm{D}^{1,2}\left(\mathbb{R}^{3}\right) \cap \mathrm{L}^{6}\left(\mathbb{R}^{3}\right)$.

\subsection{Torus Group}

We let $\mathbb{T}:=\mathbb{R} / \mathcal{T} \mathbb{Z}$ denote the torus group endowed with the canonical quotient topology. Employing the quotient mapping $\Pi: \mathbb{R} \rightarrow \mathbb{R} / \mathcal{T} \mathbb{Z}$ as a pull-back operator, we obtain a bijection between the $\mathcal{T}$-time-periodic functions $u: \mathbb{R} \rightarrow X$ and the functions $U: \mathbb{T} \rightarrow X$ defined on the torus in any setting of $X$-valued functions.

The torus $\mathbb{T}$ is further equipped with the normalized Haar-measure, which is equivalent to the normalized Lebesgue measure on $[0, \mathcal{T})$. More precisely,

$$
\forall \varphi \in C_{0}(\mathbb{T}): \quad \int_{\mathbb{T}} \varphi \mathrm{d} t:=\frac{1}{\mathcal{T}} \int_{0}^{\mathcal{T}} \varphi(t) \mathrm{d} t .
$$

For simplicity, we use $\mathrm{d} t$ to denote both the normalized Haar-measure on $\mathbb{T}$ and the Lebesgue measure on $[0, \mathcal{T})$.

We employ the Bochner spaces $\mathrm{L}^{2}\left(\mathbb{T} ; \mathrm{D}_{0, \sigma}^{1,2}\left(\mathbb{R}^{3}\right)\right)$ and $\mathrm{L}^{2}\left(\mathbb{T} ; \mathrm{D}_{0}^{-1,2}\left(\mathbb{R}^{3}\right)\right)$. We shall also need Bochner-Sobolev spaces of fractional order. We employ the Fourier transform on $\mathbb{T}$ to introduce these spaces. Recall that, for any complex Hilbert space $H$, the Fourier transform $\mathscr{F}_{\mathbb{T}}$ extends to an isomorphism

$$
\mathscr{F}_{\mathbb{T}}: \mathrm{L}^{2}(\mathbb{T} ; H) \rightarrow \mathrm{L}^{2}(\mathbb{Z} ; H), \quad \widehat{u}:=\mathscr{F}_{\mathbb{T}}(u),
$$

which is isometric with respect to the inner products in $\mathrm{L}^{2}(\mathbb{T} ; H)$ and $\mathrm{L}^{2}(\mathbb{Z} ; H)$. We define, for $\alpha \in(0, \infty)$, the space

$$
\mathrm{W}^{\alpha, 2}(\mathbb{T} ; H):=\left\{\left.u \in \mathrm{L}^{2}(\mathbb{T} ; H)|| k\right|^{\alpha} \widehat{u}(k) \in \mathrm{L}^{2}(\mathbb{Z} ; H)\right\}
$$

with the norm

$$
\|u\|_{\mathrm{W}^{\alpha, 2}(\mathbb{T} ; H)}:=\left(\sum_{k \in \mathbb{Z}}\left(1+|k|^{\alpha}\right)^{2}\|\widehat{u}(k)\|_{H}^{2}\right)^{1 / 2} .
$$


It is easy to see that $\mathrm{W}^{\alpha, 2}(\mathbb{T} ; H)$ equipped with this norm is a Hilbert space. If $H$ is a real Hilbert space, we define $\mathrm{W}^{\alpha, 2}(0, T ; H)$ and $\mathrm{W}^{\alpha, 2}(\mathbb{T} ; H)$ by using first the complexification of $H$, and subsequently restrict the elements to their real parts.

We shall introduce a weak formulation of (1) in a framework where the time axis $\mathbb{R}$ is replaced with the torus $\mathbb{T}$. To this end, we need a space of smooth solenoidal test functions of compact support in the domain $\mathbb{T} \times \mathbb{R}^{3}$. We use the quotient map

$$
\pi: \mathbb{R} \times \mathbb{R}^{3} \rightarrow \mathbb{T} \times \mathbb{R}^{3}, \quad \pi(t, x):=([t], x)
$$

to define

$$
C^{\infty}\left(\mathbb{T} \times \mathbb{R}^{3}\right):=\left\{u: \mathbb{T} \times \mathbb{R}^{3} \rightarrow \mathbb{R} \mid \exists U \in C^{\infty}\left(\mathbb{R} \times \mathbb{R}^{3}\right): U=u \circ \pi\right\} .
$$

We, then, introduce the space

$$
C_{0, \sigma}^{\infty}\left(\mathbb{T} \times \mathbb{R}^{3}\right):=\left\{u \in C^{\infty}\left(\mathbb{T} \times \mathbb{R}^{3}\right) \mid \operatorname{div} u=0, \operatorname{supp} u \text { is compact }\right\},
$$

which will serve as the space of test functions in the weak formulation.

\subsection{Time-Averaging Projection}

For a Banach space $X$, we define the projection $\mathcal{P}$ on $\mathrm{L}^{q}(\mathbb{T} ; X), q \in[1, \infty)$ by

$$
\mathcal{P}: \mathrm{L}^{q}(\mathbb{T} ; X) \rightarrow \mathrm{L}^{q}(\mathbb{T} ; X), \quad \mathcal{P} g:=\int_{\mathbb{T}} g(s) \mathrm{d} s,
$$

where the integral above is understood as a Bochner integral in $X$. Clearly, $\mathcal{P}$ is a continuous projection. Put

$$
\mathcal{P}_{\perp}: \mathrm{L}^{q}(\mathbb{T} ; X) \rightarrow \mathrm{L}^{q}(\mathbb{T} ; X), \quad \mathcal{P}_{\perp} g:=\mathrm{Id}-\mathcal{P} g .
$$

Of course, $\mathcal{P}_{\perp}$ is also a continuous projection.

Observe that $\mathcal{P} g$ time-independent. More precisely, $\mathcal{P L}^{q}(\mathbb{T} ; X)=X$. Consequently, $\mathcal{P}$ induces a decomposition

$$
\mathrm{L}^{q}(\mathbb{T} ; X)=X \oplus \mathrm{L}_{\perp}^{q}(\mathbb{T} ; X),
$$

where $\mathrm{L}_{\perp}^{q}(\mathbb{T} ; X)=\mathcal{P}_{\perp} \mathrm{L}^{q}(\mathbb{T} ; X)$. For $u \in \mathrm{L}^{q}(\mathbb{T} ; X)$, we will refer to $\mathcal{P} u$ as the steady-state part and $\mathcal{P}_{\perp} u$ as the purely oscillatory part of $u$.

\section{Existence} follows:

In the framework of the torus group, we define a weak time-periodic solution to (1) as

Definition 1. Let $f \in \mathrm{L}^{2}\left(\mathbb{T} ; \mathrm{D}_{0}^{-1,2}\left(\mathbb{R}^{3}\right)^{3}\right)$. A vector field $u \in \mathrm{L}^{2}\left(\mathbb{T} ; \mathrm{D}_{0, \sigma}^{1,2}\left(\mathbb{R}^{3}\right)\right)$ is called a weak time-periodic solution to (1) if

$$
\begin{aligned}
& \forall \Phi \in C_{0, \sigma}^{\infty}\left(\mathbb{T} \times \mathbb{R}^{3}\right): \\
& \quad \int_{\mathbb{T}} \int_{\mathbb{R}^{3}}-u \cdot \partial_{t} \Phi+\nabla u: \nabla \Phi-\lambda \partial_{1} u \cdot \Phi+(u \cdot \nabla u) \cdot \Phi \mathrm{d} x \mathrm{~d} t=\int_{\mathbb{T}}\langle f, \Phi\rangle \mathrm{d} t .
\end{aligned}
$$

Remark 1. Recalling (6), we observe for $u$ and $\Phi$, as in Definition 1, that

$$
\begin{aligned}
\int_{\mathbb{T}} \int_{\mathbb{R}^{3}}|(u \cdot \nabla u) \cdot \Phi| \mathrm{d} x \mathrm{~d} t & \leq \int_{\mathbb{T}}\|u(t)\|_{6}\|\nabla u(t)\|_{2}\|\Phi(t)\|_{3} \mathrm{~d} t \\
& \leq \sup _{t \in \mathbb{T}}\|\Phi(t)\|_{3} \int_{\mathbb{T}}\|\nabla u(t)\|_{2}^{2} \mathrm{~d} t .
\end{aligned}
$$

Thus, the integral in (13) is well-defined. 
Remark 2. In Section 5, we shall show the existence of a pressure term $\mathfrak{p}$ that, together with the weak solution, constitutes a solution $(u, \mathfrak{p})$ to $(1)$ in the sense of distributions and, thereby, justify (13) as a weak formulation. the form:

In the framework of the torus group, a slightly stronger version of Theorem 1 takes

Theorem 2. Let $f \in \mathrm{L}^{2}\left(\mathbb{T} ; \mathrm{D}_{0}^{-1,2}\left(\mathbb{R}^{3}\right)^{3}\right)$ and $\lambda \in \mathbb{R}$. There exists a weak time-periodic solution $u \in \mathrm{L}^{2}\left(\mathbb{T} ; \mathrm{D}_{0, \sigma}^{1,2}\left(\mathbb{R}^{3}\right)\right)$ to (1) that satisfies

$$
\begin{aligned}
& \mathcal{P} u \in \mathrm{D}_{0, \sigma}^{1,2}\left(\mathbb{R}^{3}\right), \\
& \mathcal{P}_{\perp} u \in \mathrm{L}^{2}\left(\mathbb{T} ; \mathrm{D}_{0, \sigma}^{1,2}\left(\mathbb{R}^{3}\right)\right) \cap \mathrm{L}^{\infty}\left(\mathbb{T} ; \mathrm{L}_{\sigma}^{2}\left(\mathbb{R}^{3}\right)\right) .
\end{aligned}
$$

Moreover,

$$
\forall \alpha \in\left[0, \frac{1}{3}\right): \quad \mathcal{P}_{\perp} u \in \mathrm{W}^{\alpha, 2}\left(\mathbb{T} ; \mathrm{L}_{\sigma}^{2}\left(\mathbb{R}^{3}\right)\right)
$$

Proof. Without loss of generalization, we assume $\lambda \geq 0$. We let $\left\{\psi_{j}\right\}_{j \in \mathbb{N}} \subset C_{0, \sigma}^{\infty}\left(\mathbb{R}^{3}\right)^{3}$ be an orthonormal basis for the Hilbert space $W_{0, \sigma}^{1,2}\left(\mathbb{R}^{3}\right)$. Since $W_{0, \sigma}^{1,2}\left(\mathbb{R}^{3}\right)$ is separable and $C_{0, \sigma}^{\infty}\left(\mathbb{R}^{3}\right)$ is dense herein, it is possible to find such a basis. For $j, k \in \mathbb{N}$ put

$$
\begin{aligned}
& \Psi_{j k}^{\#}: \mathbb{T} \times \mathbb{R}^{3} \rightarrow \mathbb{R}, \quad \Psi_{j k}^{\#}(t, x):=\frac{1}{\sqrt{2}} \psi_{j}(x) \cos \left(\frac{2 \pi}{\mathcal{T}} k t\right), \\
& \Psi_{j k}^{*}: \mathbb{T} \times \mathbb{R}^{3} \rightarrow \mathbb{R}, \quad \Psi_{j k}^{\#}(t, x):=\frac{1}{\sqrt{2}} \psi_{j}(x) \sin \left(\frac{2 \pi}{\mathcal{T}} k t\right), \\
& \Psi_{j}^{0}: \mathbb{T} \times \mathbb{R}^{3} \rightarrow \mathbb{R}, \quad \Psi_{j}^{0} k(t, x):=\psi_{j}(x) .
\end{aligned}
$$

Clearly, $\left\{\Psi_{j k}^{\#} \Psi_{j k}^{*}, \Psi_{j}^{0}\right\}_{k, j \in \mathbb{N}}$ is an orthonormal basis for $\mathrm{L}^{2}\left(\mathbb{T} ; \mathrm{W}_{0, \sigma}^{1,2}\left(\mathbb{R}^{3}\right)\right)$. For $n \geq m$, we let

$$
X_{n}^{m}:=\operatorname{span}\left\{\Psi_{j k}^{\#}, \Psi_{j k}^{*}, \Psi_{j}^{0} \mid j \leq m, k \leq n\right\}
$$

denote a finite linear span of these basis vectors. More precisely, we consider $X_{n}^{m}$ as a subspace of $\mathrm{L}^{2}\left(\mathbb{T} ; \mathrm{W}_{0, \sigma}^{1,2}\left(\mathbb{R}^{3}\right)\right)$.

We say that $U \in X_{n}^{m}$ is an approximate solution in $X_{n}^{m}$ to (1) if

$$
\begin{aligned}
& \forall \Phi \in X_{n}^{m}: \\
& \qquad \int_{\mathbb{T}} \int_{\mathbb{R}^{3}}-U \cdot \partial_{t} \Phi+\nabla U: \nabla \Phi-\lambda \partial_{1} U \cdot \Phi+(U \cdot \nabla U) \cdot \Phi \mathrm{d} x \mathrm{~d} t=\int_{\mathbb{T}}\langle f, \Phi\rangle \mathrm{d} t .
\end{aligned}
$$

We start by showing existence of such an approximate solution. Interestingly, we can use the same method that is employed in [13] (Chapter IX.4) to treat the steady-state case. Let

$$
\forall \xi=\left(\xi^{\#}, \xi^{*}, \xi^{0}\right) \in \mathbb{R}^{m \times n} \times \mathbb{R}^{m \times n} \times \mathbb{R}^{m}: \quad U_{\xi}:=\sum_{j \leq m, k \leq n} \xi_{j k}^{\#} \Psi_{j k}^{\#}+\xi_{j k}^{*} \Psi_{j k}^{*}+\xi_{j}^{0} \Psi_{j}^{0} .
$$

Define the mapping

$$
P: \mathbb{R}^{m \times n} \times \mathbb{R}^{m \times n} \times \mathbb{R}^{m} \rightarrow \mathbb{R}^{m \times n} \times \mathbb{R}^{m \times n} \times \mathbb{R}^{m}, \quad P(\xi)=\left(P^{\#}(\xi), P^{*}(\xi), P^{0}(\xi)\right)
$$


by

$$
\begin{aligned}
& P_{j k}^{\#}(\xi):=\int_{\mathbb{T}} \int_{\mathbb{R}^{3}}\left(\partial_{t} U_{\xi}-\Delta U_{\xi}-\lambda \partial_{1} U_{\xi}+U_{\xi} \cdot \nabla U_{\xi}\right) \cdot \Psi_{j k}^{\#} \mathrm{~d} x \mathrm{~d} t-\int_{\mathbb{T}}\left\langle f, \Psi_{j k}^{\#}\right\rangle \mathrm{d} t, \\
& P_{j k}^{*}(\xi):=\int_{\mathbb{T}} \int_{\mathbb{R}^{3}}\left(\partial_{t} U_{\xi}-\Delta U_{\xi}-\lambda \partial_{1} U_{\xi}+U_{\xi} \cdot \nabla U_{\xi}\right) \cdot \Psi_{j k}^{*} \mathrm{~d} x \mathrm{~d} t-\int_{\mathbb{T}}\left\langle f, \Psi_{j k}^{*}\right\rangle \mathrm{d} t, \\
& P_{j}^{0}(\xi):=\int_{\mathbb{T}} \int_{\mathbb{R}^{3}}\left(\partial_{t} U_{\xi}-\Delta U_{\xi}-\lambda \partial_{1} U_{\xi}+U_{\xi} \cdot \nabla U_{\xi}\right) \cdot \Psi_{j}^{0} \mathrm{~d} x \mathrm{~d} t-\int_{\mathbb{T}}\left\langle f, \Psi_{j}^{0}\right\rangle \mathrm{d} t .
\end{aligned}
$$

Clearly, $P$ is continuous. Moreover,

$$
\begin{aligned}
& P(\xi) \cdot \xi \\
& =\int_{\mathbb{T}} \int_{\mathbb{R}^{3}} \partial_{t} U_{\xi} \cdot U_{\xi}-\Delta U_{\xi} \cdot U_{\xi}-\lambda \partial_{1} U_{\xi} \cdot U_{\xi}+\left(U_{\xi} \cdot \nabla U_{\xi}\right) \cdot U_{\xi} \mathrm{d} x \mathrm{~d} t-\int_{\mathbb{T}}\left\langle f, U_{\xi}\right\rangle \mathrm{d} t \\
& =\int_{\mathbb{T}} \int_{\mathbb{R}^{3}}\left|\nabla U_{\xi}\right|^{2} \mathrm{~d} x \mathrm{~d} t-\int_{\mathbb{T}}\left\langle f, U_{\xi}\right\rangle \mathrm{d} t \\
& \geq\left\|U_{\xi}\right\|_{\mathrm{L}^{2}\left(\mathbb{T} ; \mathrm{D}_{0, \sigma}^{1,2}\left(\mathbb{R}^{3}\right)\right)}^{2}-\|f\|_{\mathrm{L}^{2}\left(\mathbb{T} ; \mathrm{D}_{0}^{-1,2}\left(\mathbb{R}^{3}\right)\right)}\left\|U_{\xi}\right\|_{\mathrm{L}^{2}\left(\mathbb{T} ; \mathrm{D}_{0, \sigma}^{1,2}\left(\mathbb{R}^{3}\right)\right)} .
\end{aligned}
$$

Since the family of vectors $\left\{\Psi_{j k^{\prime}}^{\#}, \Psi_{j k^{\prime}}^{*} \Psi_{j}^{0} \mid j \leq m, k \leq n\right\}$ is linearly independent in the space $\mathrm{L}^{2}\left(\mathbb{T} ; \mathrm{W}_{0, \sigma}^{1,2}\left(\mathbb{R}^{3}\right)\right)$, it is also linearly independent as a family of vectors in the space $\mathrm{L}^{2}\left(\mathbb{T} ; \mathrm{D}_{0, \sigma}^{1,2}\left(\mathbb{R}^{3}\right)\right)$. Consequently, there are constants $A, B>0$ such that

$$
\forall \xi \in \mathbb{R}^{m \times n} \times \mathbb{R}^{m \times n} \times \mathbb{R}^{m}: A|\xi| \leq\left\|U_{\xi}\right\|_{\mathrm{L}^{2}\left(\mathbb{T} ; \mathrm{D}_{0, \sigma}^{1,2}\left(\mathbb{R}^{3}\right)\right)} \leq B|\xi| .
$$

It follows that

$$
P(\xi) \cdot \xi \geq A^{2}|\xi|^{2}-B\|f\|_{\mathrm{L}^{2}\left(\mathbb{T} ; \mathrm{D}_{0}^{-1,2}\left(\mathbb{R}^{3}\right)\right)}|\xi|,
$$

whence $P(\xi) \cdot \xi>0$ for all $\xi \in \mathbb{R}^{m \times n} \times \mathbb{R}^{m \times n} \times \mathbb{R}^{m}$ with $|\xi|=2 \frac{B}{A^{2}}\|f\|$. Thus, $P$ satisfies the condition in [13] (Lemma VIII.3.1) from which the existence of $\xi_{0} \in \mathbb{R}^{m \times n} \times \mathbb{R}^{m \times n} \times \mathbb{R}^{m}$ with the property

$$
P\left(\xi_{0}\right)=0
$$

then follows. We conclude that $U_{\tilde{\xi}_{0}}$ is an approximate solution in $X_{n}^{m}$. We put $U_{n}^{m}:=U_{\tilde{\xi}_{0}}$. By a similar computation as in (18), we find

$$
0=P\left(\xi_{0}\right) \cdot \xi_{0}=\int_{\mathbb{T}} \int_{\mathbb{R}^{3}}\left|\nabla U_{n}^{m}\right|^{2} \mathrm{~d} x \mathrm{~d} t-\int_{\mathbb{T}}\left\langle f, U_{n}^{m}\right\rangle \mathrm{d} t,
$$

and, thus, conclude

$$
\left\|U_{n}^{m}\right\|_{\mathrm{L}^{2}\left(\mathbb{T} ; \mathrm{D}_{0, \sigma}^{1,2}\left(\mathbb{R}^{3}\right)\right)} \leq\|f\|_{\mathrm{L}^{2}\left(\mathbb{T} ; \mathrm{D}_{0}^{-1,2}\left(\mathbb{R}^{3}\right)\right)} .
$$

Summarizing, we have found for all $m, n \in \mathbb{N}$ an approximate solution $U_{n}^{m} \in X_{n}^{m}$ to (1) that satisfies (20).

In the next step, we seek to establish a similar bound on the norm of $\mathcal{P}_{\perp} U_{n}^{m}$ in the space $\mathrm{W}^{\alpha, 2}\left(\mathbb{T} ; \mathrm{L}_{\sigma}^{2}\left(\mathbb{R}^{3}\right)\right)$. For this purpose, it will be convenient to express $U_{n}^{m}(\cdot, x)$ in terms of the basis $\left\{\mathrm{e}^{i \frac{2 \pi}{T} k t} \mid k \in \mathbb{Z}\right\}$ of the complex Hilbert space $\mathrm{L}^{2}(\mathbb{T} ; \mathbb{C})$. More precisely, we put

$$
\forall k \in \mathbb{Z}: \quad u_{k}(x):=\int_{\mathbb{T}} U_{n}^{m}(t, x) \mathrm{e}^{-i \frac{2 \pi}{T} k t} \mathrm{~d} t \in C_{0, \sigma}^{\infty}\left(\mathbb{R}^{3}\right) .
$$


Clearly, $u_{k}=0$ for all $|k|>n$. Since $\left\{u_{k}(x) \mid k \in \mathbb{Z}\right\}$ are the Fourier coefficients of $U_{n}^{m}(\cdot, x)$, we have

$$
U_{n}^{m}(t, x)=\sum_{k \in \mathbb{Z}} u_{k}(x) \mathrm{e}^{i \frac{2 \pi}{T} k t}=\sum_{|k| \leq n} u_{k}(x) \mathrm{e}^{i \frac{2 \pi}{T} k t}
$$

Observe that

$$
\begin{aligned}
& \mathcal{P} U_{n}^{m}(t, x)=u_{0}(x), \\
& \mathcal{P}_{\perp} U_{n}^{m}(t, x)=\sum_{k \in \mathbb{Z} \backslash\{0\}} u_{k}(x) \mathrm{e}^{i \frac{2 \pi}{T} k t}=\sum_{|k| \leq n, k \neq 0} u_{k}(x) \mathrm{e}^{i \frac{2 \pi}{T} k t} .
\end{aligned}
$$

For any $l \in \mathbb{Z} \backslash\{0\}$ and $\gamma \in \mathbb{R}, \gamma>0$ we will now use $(-i) \operatorname{sgn}(l)|l|^{-\gamma} \overline{u_{l}} \mathrm{e}^{-i \frac{2 \pi}{T} l t}$ as a test function in the weak formulation (17). We, therefore, compute

$$
\begin{aligned}
\int_{\mathbb{T}} \int_{\mathbb{R}^{3}} \partial_{t} U_{n}^{m} \cdot(-i) \operatorname{sgn}(l)|l|^{-\gamma} \overline{u_{l}} \mathrm{e}^{-i \frac{2 \pi}{T} l t} \mathrm{~d} x \mathrm{~d} t \\
\quad=\int_{\mathbb{T}} \int_{\mathbb{R}^{3}}\left(\sum_{k \in \mathbb{Z}} i \frac{2 \pi}{\mathcal{T}} k u_{k}(x) \mathrm{e}^{i \frac{2 \pi}{T} k t}\right) \cdot(-i) \operatorname{sgn}(l)|l|^{-\gamma} \overline{u_{l}} \mathrm{e}^{-i \frac{2 \pi}{T} l t} \mathrm{~d} x \mathrm{~d} t \\
=\int_{\mathbb{R}^{3}} \frac{2 \pi}{\mathcal{T}} l \operatorname{sgn}(l)|l|^{-\gamma} u_{l} \overline{u_{l}} \mathrm{~d} x \\
=\frac{2 \pi}{\mathcal{T}}|l|^{1-\gamma} \int_{\mathbb{R}^{3}}\left|u_{l}\right|^{2} \mathrm{~d} x .
\end{aligned}
$$

Similarly, we compute

$$
\begin{aligned}
\int_{\mathbb{T}} \int_{\mathbb{R}^{3}} \nabla U_{n}^{m}: \nabla\left[(-i) \operatorname{sgn}(l)|l|^{-\gamma} \overline{u_{l}} \mathrm{e}^{\left.-i \frac{2 \pi}{T} l t\right]} \mathrm{d} x \mathrm{~d} t\right. & \\
& =(-i) \operatorname{sgn}(l)|l|^{-\gamma} \int_{\mathbb{R}^{3}}\left|\nabla u_{l}\right|^{2} \mathrm{~d} x,
\end{aligned}
$$

and

$$
\int_{\mathbb{T}} \int_{\mathbb{R}^{3}} \lambda \partial_{1} U_{n}^{m} \cdot(-i) \operatorname{sgn}(l)|l|^{-\gamma} \overline{u_{l}} \mathrm{e}^{-i \frac{2 \pi}{T} l t} \mathrm{~d} x \mathrm{~d} t=(-i) \operatorname{sgn}(l)|l|^{-\gamma} \lambda \int_{\mathbb{R}^{3}} \partial_{1} u_{l} \overline{u_{l}} \mathrm{~d} x .
$$

Since $U_{n}^{m}$ is a real function, it follows that $\overline{u_{k}}=u_{-k}$. Consequently,

$$
\begin{aligned}
& \int_{\mathbb{T}} \int_{\mathbb{R}^{3}}\left(U_{n}^{m} \cdot \nabla U_{n}^{m}\right) \cdot(-i) \operatorname{sgn}(l)|l|^{-\gamma} \overline{u_{l}} \mathrm{e}^{-i \frac{2 \pi}{T} l t} \mathrm{~d} x \mathrm{~d} t \\
& =\int_{\mathbb{T}} \int_{\mathbb{R}^{3}}\left(\left(\sum_{|k| \leq n} u_{k}(x) \mathrm{e}^{i \frac{2 \pi}{T} k t}\right) \cdot \nabla\left(\sum_{|h| \leq n} u_{h}(x) \mathrm{e}^{i \frac{2 \pi}{T} h t}\right)\right) \\
& =\int_{\mathbb{T}} \int_{\mathbb{R}^{3}} \sum_{|k|,|h| \leq n}\left(u_{k} \cdot \nabla u_{h}\right) \cdot\left((-i) \operatorname{sgn}(l)|l|^{-\gamma} \overline{u_{l}} \mathrm{e}^{-i \frac{2 \pi}{T} l t} \mathrm{~d} x \mathrm{~d} t\right. \\
& =(-i) \operatorname{sgn}(l)|l|^{-\gamma} \int_{\mathbb{R}^{3}} \sum_{|k| \leq n}\left(u_{k} \cdot \nabla u_{l-l}\right) \cdot \mathrm{e}_{-l} \mathrm{~d} x .
\end{aligned}
$$


For $|l| \leq n$, it is easy to see that the function $(-i) \operatorname{sgn}(l)|l|^{-\gamma} \overline{u_{l}} \mathrm{e}^{-i \frac{2 \pi}{T} l t}$ belongs to the complexification $X_{n}^{m} \oplus i X_{n}^{m}$ of $X_{n}^{m}$. Consequently, we can use it as a test function in the weak formulation (17). From (17) and (24)-(27) it, therefore, follows that

$$
\begin{aligned}
\frac{2 \pi}{\mathcal{T}}|l|^{1-\gamma} \int_{\mathbb{R}^{3}}\left|u_{l}\right|^{2} \mathrm{~d} x= & -(-i) \operatorname{sgn}(l)|l|^{-\gamma} \int_{\mathbb{R}^{3}}\left|\nabla u_{l}\right|^{2} \mathrm{~d} x \\
& +(-i) \operatorname{sgn}(l)|l|^{-\gamma} \lambda \int_{\mathbb{R}^{3}} \partial_{1} u_{l} \overline{u_{l}} \mathrm{~d} x \\
& -(-i) \operatorname{sgn}(l)|l|^{-\gamma} \int_{\mathbb{R}^{3}} \sum_{|k| \leq n}\left(u_{k} \cdot \nabla u_{l-k}\right) \cdot u_{-l} \mathrm{~d} x \\
& +(-i) \operatorname{sgn}(l)|l|^{-\gamma} \int_{\mathbb{T}}\left\langle f, \overline{u_{l}}\right\rangle \mathrm{e}^{-i \frac{2 \pi}{T} l t} \mathrm{~d} t .
\end{aligned}
$$

We shall estimate the terms on the right-hand side above. To estimate the second term, we employ first Hölder's and then Young's inequality to obtain

$$
\begin{aligned}
\left.|| l\right|^{-\gamma} \lambda \int_{\mathbb{R}^{3}} \partial_{1} u_{l} \overline{u_{l}} \mathrm{~d} x \mid & \leq|l|^{-\gamma} \lambda\left(\int_{\mathbb{R}^{3}}\left|\nabla u_{l}\right|^{2} \mathrm{~d} x\right)^{\frac{1}{2}}\left(\int_{\mathbb{R}^{3}}\left|u_{l}\right|^{2} \mathrm{~d} x\right)^{\frac{1}{2}} \\
& \leq|l|^{-\gamma}\left(\frac{1}{2} \frac{2 \pi}{\mathcal{T}} \int_{\mathbb{R}^{3}}\left|u_{l}\right|^{2} \mathrm{~d} x+c_{1} \int_{\mathbb{R}^{3}}\left|\nabla u_{l}\right|^{2} \mathrm{~d} x\right) \\
& \leq|l|^{1-\gamma} \frac{1}{2} \frac{2 \pi}{\mathcal{T}} \int_{\mathbb{R}^{3}}\left|u_{l}\right|^{2} \mathrm{~d} x+c_{1} \int_{\mathbb{R}^{3}}\left|\nabla u_{l}\right|^{2} \mathrm{~d} x,
\end{aligned}
$$

with a constant $c_{1}=c_{1}(\lambda, \mathcal{T})>0$ according to Young's inequality. Recalling (6), we estimate, employing Hölder's inequality, the third term on the right-hand side in (28) by

$$
\begin{aligned}
&\left.|| l\right|^{-\gamma} \int_{\mathbb{R}^{3}} \sum_{|k| \leq n}\left(u_{k} \cdot \nabla u_{l-k}\right) \cdot u_{-l} \mathrm{~d} x \mid \\
& \leq \leq\left. l\right|^{-\gamma} \sum_{|k| \leq n}\left(\int_{\mathbb{R}^{3}}\left|u_{k}\right|^{6} \mathrm{~d} x\right)^{\frac{1}{6}}\left(\int_{\mathbb{R}^{3}}\left|\nabla u_{l-k}\right|^{2} \mathrm{~d} x\right)^{\frac{1}{2}}\left(\int_{\mathbb{R}^{3}}\left|u_{l}\right|^{3} \mathrm{~d} x\right)^{\frac{1}{3}} \\
& \leq c_{2}|l|^{-\gamma} \sum_{|k| \leq n}\left(\int_{\mathbb{R}^{3}}\left|\nabla u_{k}\right|^{2} \mathrm{~d} x\right)^{\frac{1}{2}}\left(\int_{\mathbb{R}^{3}}\left|\nabla u_{l-k}\right|^{2} \mathrm{~d} x\right)^{\frac{1}{2}}\left(\int_{\mathbb{R}^{3}}\left|u_{l}\right|^{3} \mathrm{~d} x\right)^{\frac{1}{3}} \\
& \leq c_{2}|l|^{-\gamma}\left(\int_{\mathbb{R}^{3}}\left|u_{l}\right|^{3} \mathrm{~d} x\right)^{\frac{1}{3}}\left(\sum_{|k| \leq n} \int_{\mathbb{R}^{3}}\left|\nabla u_{k}\right|^{2} \mathrm{~d} x\right)^{\frac{1}{2}}\left(\sum_{|k| \leq n} \int_{\mathbb{R}^{3}}\left|\nabla u_{l-k}\right|^{2} \mathrm{~d} x\right)^{\frac{1}{2}},
\end{aligned}
$$

with a constant $c_{2}>0$ according to the embedding (6). Employing Lemma 1 to estimate the first integral on the right-hand side above, we further obtain

$$
\begin{aligned}
\left.|| l\right|^{-\gamma} \int_{\mathbb{R}^{3}} \sum_{|k| \leq n}\left(u_{l-k} \cdot \nabla u_{k}\right) \cdot u_{-l} \mathrm{~d} x \mid \\
\quad \leq c_{3}|l|^{-\gamma}\left(\int_{\mathbb{R}^{3}}\left|u_{l}\right|^{2} \mathrm{~d} x\right)^{\frac{1}{4}}\left(\int_{\mathbb{R}^{3}}\left|\nabla u_{l}\right|^{2} \mathrm{~d} x\right)^{\frac{1}{4}}\left(\sum_{k \in \mathbb{Z}} \int_{\mathbb{R}^{3}}\left|\nabla u_{k}\right|^{2} \mathrm{~d} x\right),
\end{aligned}
$$

whence, by Plancherel's equality,

$$
\begin{aligned}
&\left.|| l\right|^{-\gamma} \int_{\mathbb{R}^{3}} \sum_{|k| \leq n}\left(u_{k} \cdot \nabla u_{l-k}\right) \cdot u_{-l} \mathrm{~d} x \mid \\
& \quad \leq c_{4}|l|^{-\gamma}\left(\int_{\mathbb{R}^{3}}\left|u_{l}\right|^{2} \mathrm{~d} x\right)^{\frac{1}{4}}\left(\int_{\mathbb{R}^{3}}\left|\nabla u_{l}\right|^{2} \mathrm{~d} x\right)^{\frac{1}{4}}\left(\int_{\mathbb{T}} \int_{\mathbb{R}^{3}}\left|\nabla U_{n}^{m}\right|^{2} \mathrm{~d} x \mathrm{~d} t\right) .
\end{aligned}
$$


We can estimate the last term on the right-hand side in (28) by

$$
\left.|| l\right|^{-\gamma} \int_{\mathbb{T}}\left\langle f, \overline{u_{l}}\right\rangle \mathrm{e}^{-i \frac{2 \pi}{T} l t} \mathrm{~d} t \mid \leq\left\|f_{l}\right\|_{\mathrm{D}_{0}^{-1,2}\left(\mathbb{R}^{3}\right)}^{2}+\left\|\nabla u_{l}\right\|_{2}^{2},
$$

where $f_{l}$ denotes the $l^{\prime}$ th Fourier coefficient of $f$. Collecting (28)-(31) and recalling (20), we can now deduce

$$
\begin{aligned}
& |l|^{1-\gamma} \int_{\mathbb{R}^{3}}\left|u_{l}\right|^{2} \mathrm{~d} x \\
& \quad \leq c_{5}\left(\int_{\mathbb{R}^{3}}\left|\nabla u_{l}\right|^{2} \mathrm{~d} x+|l|^{-\gamma}\left(\int_{\mathbb{R}^{3}}\left|u_{l}\right|^{2} \mathrm{~d} x\right)^{\frac{1}{4}}\left(\int_{\mathbb{R}^{3}}\left|\nabla u_{l}\right|^{2} \mathrm{~d} x\right)^{\frac{1}{4}}+\left\|f_{l}\right\|_{\mathrm{D}_{0}^{-1,2}\left(\mathbb{R}^{3}\right)}^{2}\right),
\end{aligned}
$$

where $c_{5}=c_{5}(\|f\|, \mathcal{T}, \lambda)$. Finally, we employ Young's inequality to estimate

$$
\begin{aligned}
|l|^{-\gamma}\left(\int_{\mathbb{R}^{3}}\left|u_{l}\right|^{2} \mathrm{~d} x\right)^{\frac{1}{4}} & \left(\int_{\mathbb{R}^{3}}\left|\nabla u_{l}\right|^{2} \mathrm{~d} x\right)^{\frac{1}{4}} \\
& =|l|^{-\gamma-\frac{1}{4}(1-\gamma)}\left(\int_{\mathbb{R}^{3}}|l|^{1-\gamma}\left|u_{l}\right|^{2} \mathrm{~d} x\right)^{\frac{1}{4}}\left(\int_{\mathbb{R}^{3}}\left|\nabla u_{l}\right|^{2} \mathrm{~d} x\right)^{\frac{1}{4}} \\
& \leq c_{6}|l|^{-2\left(\frac{3}{4} \gamma+\frac{1}{4}\right)}+\frac{1}{2 c_{5}} \int_{\mathbb{R}^{3}}|l|^{1-\gamma}\left|u_{l}\right|^{2} \mathrm{~d} x+c_{7} \int_{\mathbb{R}^{3}}\left|\nabla u_{l}\right|^{2} \mathrm{~d} x
\end{aligned}
$$

and conclude

$$
|l|^{1-\gamma} \int_{\mathbb{R}^{3}}\left|u_{l}\right|^{2} \mathrm{~d} x \leq c_{8}\left(\int_{\mathbb{R}^{3}}\left|\nabla u_{l}\right|^{2} \mathrm{~d} x+|l|^{-2\left(\frac{3}{4} \gamma+\frac{1}{4}\right)}+\left\|f_{l}\right\|_{\mathrm{D}_{0}^{-1,2}\left(\mathbb{R}^{3}\right)}^{2}\right) .
$$

We have derived the above inequality for $l \in \mathbb{Z} \backslash\{0\}$ with $|l| \leq n$. However, since $u_{l}=0$ for $|l|>n$, the above inequality holds for all $l \in \mathbb{Z} \backslash\{0\}$. We will now sum both sides over l. For $\gamma>\frac{1}{3}$, we have

$$
-2\left(\frac{3}{4} \gamma+\frac{1}{4}\right)<-1
$$

whence, using again Plancherel's equality, it follows that

$$
\begin{aligned}
\sum_{l \in \mathbb{Z} \backslash\{0\}}|l|^{1-\gamma}\left\|u_{l}\right\|_{\mathrm{L}^{2}\left(\mathbb{R}^{3}\right)}^{2} \mathrm{~d} x & \leq c_{9}\left(\sum_{l \in \mathbb{Z}} \int_{\mathbb{R}^{3}}\left|\nabla u_{l}\right|^{2} \mathrm{~d} x+1+\|f\|_{\mathrm{L}^{2}\left(\mathbb{T} ; \mathrm{D}_{0}^{-1,2}\left(\mathbb{R}^{3}\right)\right)}^{2}\right) \\
& =c_{9}\left(\int_{\mathbb{T}} \int_{\mathbb{R}^{3}}\left|\nabla u_{n}^{m}\right|^{2} \mathrm{~d} x \mathrm{~d} t+1+\|f\|_{\mathrm{L}^{2}\left(\mathbb{T} ; \mathrm{D}_{0}^{-1,2}\left(\mathbb{R}^{3}\right)\right)}^{2}\right),
\end{aligned}
$$

where $c_{9}=c_{9}(\|f\|, \gamma, \mathcal{T}, \lambda)$. Recalling (20), we deduce

$$
\forall \gamma \in\left(\frac{1}{3}, \infty\right): \quad \sum_{l \in \mathbb{Z} \backslash\{0\}}|l|^{1-\gamma}\left\|u_{l}\right\|_{\mathrm{L}^{2}\left(\mathbb{R}^{3}\right)}^{2} \mathrm{~d} x \leq c_{9},
$$

with $c_{9}$ independent on $m$ and $n$. Recalling (23), we conclude for all $\alpha \in\left[0, \frac{1}{3}\right)$ that $\mathcal{P}_{\perp} U_{n}^{m} \in$ $\mathrm{W}^{\alpha, 2}\left(\mathbb{T} ; \mathrm{L}_{\sigma}^{2}\left(\mathbb{R}^{3}\right)\right)$ with

$$
\forall \alpha \in\left[0, \frac{1}{3}\right): \quad\left\|\mathcal{P}_{\perp} U_{n}^{m}\right\|_{\mathrm{W}^{\alpha, 2}\left(\mathbb{T} ; \mathrm{L}_{\sigma}^{2}\left(\mathbb{R}^{3}\right)\right)} \leq c_{10},
$$

where $c_{10}=c_{10}(\alpha,\|f\|, \mathcal{T}, \lambda)$ is independent on $m$ and $n$.

In the next step of the proof, we wish to establish an estimate of the approximate solution in the $L^{\infty}\left(\mathbb{T} ; L^{2}\left(\mathbb{R}^{3}\right)^{3}\right)$-norm. For this purpose, we choose again an appropriate 
test function and utilize that $U_{n}^{m}$ satisfies (17). We shall also need to pass to the limit $n \rightarrow \infty$.

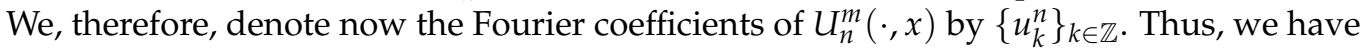

$$
U_{n}^{m}(t, x)=\sum_{k \in \mathbb{Z}} u_{k}^{n}(x) \mathrm{e}^{i \frac{2 \pi}{T} k t}=\sum_{|k| \leq n} u_{k}^{n}(x) \mathrm{e}^{i \frac{2 \pi}{T} k t}
$$

We choose for $j, l \in \mathbb{Z}$ with $|j|,|l| \leq n$ the function $u_{j}^{n} \mathrm{e}^{-i \frac{2 \pi}{T} l t} \in X_{n}^{m} \oplus i X_{n}^{m}$ as a test function in (17) and obtain

$$
\begin{aligned}
\int_{\mathbb{T}} \int_{\mathbb{R}^{3}}- & U_{n}^{m} \cdot\left(-i \frac{2 \pi}{\mathcal{T}} l u_{j}^{n} \mathrm{e}^{-i \frac{2 \pi}{T} l t}\right)+\nabla U_{n}^{m}: \nabla u_{j}^{n} \mathrm{e}^{-i \frac{2 \pi}{T} l t} \\
& \quad-\lambda \partial_{1} U_{n}^{m} \cdot u_{j}^{n} \mathrm{e}^{-i \frac{2 \pi}{\mathcal{T}} l t}+\left(U_{n}^{m} \cdot \nabla U_{n}^{m}\right) \cdot u_{j}^{n} \mathrm{e}^{-i \frac{2 \pi}{\mathcal{T}} l t} \mathrm{~d} x \mathrm{~d} t=\int_{\mathbb{T}}\left\langle f, u_{j}^{n}\right\rangle \mathrm{e}^{-i \frac{2 \pi}{\mathcal{T}} l t} \mathrm{~d} t .
\end{aligned}
$$

Inserting the Fourier series (35) for $U_{n}^{m}$ in the above identity, we find

$$
\begin{aligned}
\int_{\mathbb{R}^{3}} i \frac{2 \pi}{\mathcal{T}} l u_{l}^{n} u_{j}^{n}+\nabla u_{l}^{n}: \nabla u_{j}^{n}-\lambda \partial_{1} u_{l}^{n} \cdot u_{j}^{n}+\sum_{|k| \leq n}\left(u_{l-k}^{n} \cdot \nabla u_{k}^{n}\right) \cdot u_{j}^{n} \mathrm{~d} x & \\
& =\int_{\mathbb{T}}\left\langle f, u_{j}^{n}\right\rangle \mathrm{e}^{-i \frac{2 \pi}{\mathcal{T}} l s} \mathrm{~d} s .
\end{aligned}
$$

Multiplying both sides above with $\mathrm{e}^{i \frac{2 \pi}{T}(l+j) t}$ and summing over $j$ and $l$, we further deduce

$$
\begin{aligned}
\sum_{\substack{|j|,|l| \leq n \\
j \neq 0}} \int_{\mathbb{R}^{3}} i \frac{2 \pi}{\mathcal{T}} l u_{l}^{n} u_{j}^{n} \mathrm{e}^{i \frac{2 \pi}{\mathcal{T}}(l+j) t} \mathrm{~d} x= & \sum_{\substack{|j|,|l| \leq n \\
j \neq 0}} \int_{\mathbb{R}^{3}}-\nabla u_{l}^{n}: \nabla u_{j}^{n} \mathrm{e}^{i \frac{2 \pi}{\mathcal{T}}(l+j) t} \mathrm{~d} x \\
& +\sum_{\substack{|j|,|l| \leq n \\
j \neq 0}} \int_{\mathbb{R}^{3}} \lambda \partial_{1} u_{l}^{n} \cdot u_{j}^{n} \mathrm{e}^{i \frac{2 \pi}{\mathcal{T}}(l+j) t} \mathrm{~d} x \\
& -\sum_{\substack{|k|,|j|,|l| \leq n \\
j \neq 0}} \int_{\mathbb{R}^{3}}\left(u_{l-k}^{n} \cdot \nabla u_{k}^{n}\right) \cdot u_{j}^{n} \mathrm{e}^{i \frac{2 \pi}{\mathcal{T}}(l+j) t} \mathrm{~d} x \\
& +\sum_{\substack{|j|,|l| \leq n \\
j \neq 0}} \int_{\mathbb{T}}\left\langle f, u_{j}^{n}\right\rangle \mathrm{e}^{-i \frac{2 \pi}{\mathcal{T}} l s} \mathrm{~d} s \mathrm{e}^{i \frac{2 \pi}{\mathcal{T}}(l+j) t} .
\end{aligned}
$$

In the sums in (36), we recognize familiar quantities. For example, we compute, recalling that $\mathcal{P}_{\perp} U_{n}^{m}$ is a real function,

$$
\begin{aligned}
\partial_{t} \int_{\mathbb{R}^{3}}\left|\mathcal{P}_{\perp} U_{n}^{m}(t, x)\right|^{2} \mathrm{~d} x= & \partial_{t} \int_{\mathbb{R}^{3}} \sum_{\substack{|l|,|j| \leq n \\
l, j \neq 0}} u_{l}^{n} u_{j}^{n} \mathrm{e}^{i \frac{2 \pi}{\mathcal{T}}(l+j) t} \mathrm{~d} x \\
= & \sum_{\substack{|l|,|j| \leq n \\
l, j \neq 0}} \int_{\mathbb{R}^{3}} i \frac{2 \pi}{\mathcal{T}}(l+j) u_{l}^{n} u_{j}^{n} \mathrm{e}^{i \frac{2 \pi}{\mathcal{T}}(l+j) t} \mathrm{~d} x \\
= & \sum_{\substack{|l|,|j| \leq n \\
j \neq 0}} \int_{\mathbb{R}^{3}} i \frac{2 \pi}{\mathcal{T}} l u_{l}^{n} u_{j}^{n} \mathrm{e}^{i \frac{2 \pi}{\mathcal{T}}(l+j) t} \mathrm{~d} x \\
& +\sum_{\substack{|l|,|j| \leq n \\
l \neq 0}} \int_{\mathbb{R}^{3}} i \frac{2 \pi}{\mathcal{T}} j u_{l}^{n} u_{j}^{n} \mathrm{e}^{i \frac{2 \pi}{\mathcal{T}}(l+j) t} \mathrm{~d} x \\
= & 2 \sum_{\substack{|l|,|j| \leq n \\
j \neq 0}} \int_{\mathbb{R}^{3}} i \frac{2 \pi}{\mathcal{T}} l u_{l}^{n} u_{j}^{n} \mathrm{e}^{i \frac{2 \pi}{\mathcal{T}}(l+j) t} \mathrm{~d} x .
\end{aligned}
$$


We have, hereby, identified the left-hand side in (36) with $\frac{1}{2} \partial_{t} \int_{\mathbb{R}^{3}}\left|\mathcal{P}_{\perp} U^{m}(t, x)\right|^{2} \mathrm{~d} x$. Similar identifications can be made for the terms on the right-hand side as well. We immediately see that

$$
\sum_{\substack{|l|,|j| \leq n \\ j \neq 0}} \int_{\mathbb{R}^{3}} \nabla u_{l}^{n}: \nabla u_{j}^{n} \mathrm{e}^{-i \frac{2 \pi}{T}(l+j) t} \mathrm{~d} x=\int_{\mathbb{R}^{3}} \nabla U_{n}^{m}: \nabla \mathcal{P}_{\perp} U_{n}^{m} \mathrm{~d} x
$$

and

$$
\sum_{\substack{|l|,|j| \leq n \\ j \neq 0}} \int_{\mathbb{R}^{3}} \lambda \partial_{1} u_{l}^{n} \cdot u_{j}^{n} \mathrm{e}^{-i \frac{2 \pi}{T}(l+j) t} \mathrm{~d} x=\int_{\mathbb{R}^{3}} \lambda \partial_{1} U_{n}^{m} \cdot \mathcal{P}_{\perp} U_{n}^{m} \mathrm{~d} x .
$$

We also see that

$$
\sum_{\substack{|l|,|j| \leq n \\ j \neq 0}} \int_{\mathbb{T}}\left\langle f, u_{j}^{n}\right\rangle \mathrm{e}^{-i \frac{2 \pi}{T} l s} \mathrm{~d} s \mathrm{e}^{i \frac{2 \pi}{T}(l+j) t}=\sum_{|l| \leq n}\left\langle f_{l}, \mathcal{P}_{\perp} U_{n}^{m}\right\rangle \mathrm{e}^{i \frac{2 \pi}{T} l t},
$$

where $f_{l}$ denotes the $l^{\prime}$ th Fourier coefficient of $f$. Combining (36)-(40), we conclude that

$$
\begin{aligned}
\frac{1}{2} \partial_{t} \int_{\mathbb{R}^{3}}\left|\mathcal{P}_{\perp} U_{n}^{m}(t, x)\right|^{2} \mathrm{~d} x= & -\int_{\mathbb{R}^{3}} \nabla U_{n}^{m}: \nabla \mathcal{P}_{\perp} U_{n}^{m} \mathrm{~d} x+\int_{\mathbb{R}^{3}} \lambda \partial_{1} U_{n}^{m} \cdot \mathcal{P}_{\perp} U_{n}^{m} \mathrm{~d} x \\
& -\sum_{\substack{|k||j|,|l| \leq n \\
j \neq 0}} \int_{\mathbb{R}^{3}}\left(u_{l-k}^{n} \cdot \nabla u_{k}^{n}\right) \cdot u_{j}^{n} \mathrm{e}^{i \frac{2 \pi}{T}(l+j) t} \mathrm{~d} x \\
& +\sum_{|l| \leq n}\left\langle f_{l}, \mathcal{P}_{\perp} U_{n}^{m}\right\rangle \mathrm{e}^{i \frac{2 \pi}{T} l t} .
\end{aligned}
$$

Observe that we now have a pointwise identity for $\partial_{t} \int_{\mathbb{R}^{3}}\left|\mathcal{P}_{\perp} U_{n}^{m}(t, x)\right|^{2} \mathrm{~d} x$. To capitalize on this information, we compute for $h \in \mathbb{Z}$ the $h^{\prime}$ th Fourier coefficient on both sides in (41) to find that

$$
\begin{aligned}
\int_{\mathbb{T}}\left(-i \frac{2 \pi}{\mathcal{T}} h\right)\left(\frac{1}{2} \int_{\mathbb{R}^{3}}\left|\mathcal{P}_{\perp} U_{n}^{m}(t, x)\right|^{2} \mathrm{~d} x\right) \mathrm{e}^{-i \frac{2 \pi}{T} h t} \mathrm{~d} t \\
=\int_{\mathbb{T}}\left(\int_{\mathbb{R}^{3}}-\nabla U_{n}^{m}: \nabla \mathcal{P}_{\perp} U_{n}^{m} \mathrm{~d} x+\lambda \partial_{1} U_{n}^{m} \cdot \mathcal{P}_{\perp} U_{n}^{m} \mathrm{~d} x\right) \mathrm{e}^{-i \frac{2 \pi}{T} h t} \mathrm{~d} t \\
\quad-\sum_{\substack{|k|,|j| \leq n \\
j \neq 0,|h-j| \leq n}} \int_{\mathbb{R}^{3}}\left(u_{h-j-k}^{n} \cdot \nabla u_{k}^{n}\right) \cdot u_{j}^{n} \mathrm{~d} x+\left\langle f_{h}, \mathcal{P}_{\perp} U_{n}^{m}\right\rangle .
\end{aligned}
$$

For the purpose of expressing the sum on the right-hand side above in terms of more well-known quantities, we shall pass to the limit $n \rightarrow \infty$. First, we put

$$
Y^{m}:=\operatorname{span}\left\{\psi_{j} \mid j \leq m\right\} \subset \mathrm{W}_{0, \sigma}^{1,2}\left(\mathbb{R}^{3}\right) .
$$

Since $Y^{m}$ is a finite dimensional vector space, all norms on $Y^{m}$ are equivalent. From (20) and (34), we, thus, deduce that $\left\{U_{n}^{m}\right\}_{n=1}^{\infty}$ is bounded in the space $\mathrm{L}^{2}\left(\mathbb{T} ; Y^{m}\right)$ and $\left\{\mathcal{P}_{\perp} U_{n}^{m}\right\}_{n=1}^{\infty}$ in $\mathrm{W}^{\gamma, 2}\left(\mathbb{T} ; Y^{m}\right)$ for all $\gamma \in\left[0, \frac{1}{3}\right)$. Consequently, there is a $U^{m} \in \mathrm{L}^{2}\left(\mathbb{T} ; Y^{m}\right)$ with $\mathcal{P}_{\perp} U^{m} \in W^{\gamma, 2}\left(\mathbb{T} ; Y^{m}\right)$ and a subsequence of $\left\{U_{n}^{m}\right\}_{n=1}^{\infty}$, which, for simplicity, we still denote by $\left\{U_{n}^{m}\right\}_{n=1}^{\infty}$, such that

$$
\begin{aligned}
& U_{n}^{m} \rightarrow U^{m} \text { in } \mathrm{L}^{2}\left(\mathbb{T} ; Y^{m}\right) \text { as } n \rightarrow \infty, \\
\forall \gamma \in\left[0, \frac{1}{3}\right): & \mathcal{P}_{\perp} U_{n}^{m} \rightarrow \mathcal{P}_{\perp} U^{m} \text { in } \mathrm{W}^{\gamma, 2}\left(\mathbb{T} ; Y^{m}\right) \text { as } n \rightarrow \infty .
\end{aligned}
$$

It follows from (43) that $\mathcal{P} U_{n}^{m} \rightarrow \mathcal{P} U^{m}$ in $Y^{m}$, which, since $Y^{m}$ is finite dimensional, implies strong convergence $\mathcal{P} U_{n}^{m} \rightarrow \mathcal{P} U^{m}$ in $Y^{m}$ as $n \rightarrow \infty$. Moreover, again due to the 
fact that $Y^{m}$ is finite dimensional, $W^{\gamma_{1}, 2}\left(\mathbb{T} ; Y^{m}\right)$ is compactly embedded in $W^{\gamma_{2}, 2}\left(\mathbb{T} ; Y^{m}\right)$ for $\gamma_{1}>\gamma_{2} \geq 0$. Consequently, (44) implies strong convergence $\mathcal{P}_{\perp} U_{n}^{m} \rightarrow \mathcal{P}_{\perp} U^{m}$ in $\mathrm{W}^{\gamma, 2}\left(\mathbb{T} ; Y^{m}\right)$ as $n \rightarrow \infty$ for every $\gamma \in[0,1 / 3)$. We, thus, have

$$
\forall \gamma \in\left[0, \frac{1}{3}\right): U_{n}^{m} \rightarrow U^{m} \text { in } \mathrm{W}^{\gamma, 2}\left(\mathbb{T} ; Y^{m}\right) \text { as } n \rightarrow \infty .
$$

Finally, in view of (20) and (34), we have

$$
\forall \gamma \in\left[0, \frac{1}{3}\right): \quad\left\|U^{m}\right\|_{\mathrm{L}^{2}\left(\mathbb{T} ; \mathrm{D}_{0, \sigma}^{1,2}\left(\mathbb{R}^{3}\right)\right)}+\left\|\mathcal{P}_{\perp} U^{m}\right\|_{\mathrm{W}^{\gamma, 2}\left(\mathbb{T} ; \mathrm{L}^{2}\left(\mathbb{R}^{3}\right)^{3}\right)} \leq c_{11}
$$

with a constant $c_{11}>0$ independent on $m$. We shall now pass to the limit $n \rightarrow \infty$ in (42). We start by verifying that

$$
\begin{aligned}
\lim _{n \rightarrow \infty} \sum_{\substack{|k|,|j| \leq n \\
j \neq 0,|h-j| \leq n}} \int_{\mathbb{R}^{3}}\left(u_{h-j-k}^{n} \cdot \nabla u_{k}^{n}\right) \cdot u_{j}^{n} \mathrm{~d} x & \\
& =\int_{\mathbb{T}}\left(\int_{\mathbb{R}^{3}} U^{m} \cdot \nabla U^{m} \cdot \mathcal{P}_{\perp} U^{m} \mathrm{~d} x\right) \mathrm{e}^{-i \frac{2 \pi}{T} h t} \mathrm{~d} t .
\end{aligned}
$$

Observe that the right-hand side of (47) is well-defined since $W^{\frac{1}{4}, 2}\left(\mathbb{T} ; Y^{m}\right)$ is embedded in $\mathrm{L}^{4}\left(\mathbb{T} ; Y^{m}\right)$ and, thus, $U^{m}, \nabla U^{m} \in \mathrm{L}^{4}\left(\mathbb{T} ; Y^{m}\right)$. In fact, letting $\left\{u_{k}\right\}_{k \in \mathbb{Z}} \subset Y^{m}$ denote the Fourier coefficients of $U^{m}$, we have the identity

$$
\int_{\mathbb{T}}\left(\int_{\mathbb{R}^{3}} U^{m} \cdot \nabla U^{m} \cdot \mathcal{P}_{\perp} U^{m} \mathrm{~d} x\right) \mathrm{e}^{-i \frac{2 \pi}{T} h t} \mathrm{~d} t=\sum_{\substack{k, j \in \mathbb{Z} \\ j \neq 0}} \int_{\mathbb{R}^{3}}\left(u_{h-j-k} \cdot \nabla u_{k}\right) \cdot u_{j} \mathrm{~d} x .
$$

We further observe, recalling that $u_{k}^{n}=0$ for $|k|>n$, that

$$
\begin{aligned}
& \sum_{\substack{|k|,|j| \leq n \\
j \neq 0,|h-j| \leq n}} \int_{\mathbb{R}^{3}}\left(u_{h-j-k}^{n} \cdot \nabla u_{k}^{n}\right) \cdot u_{j}^{n} \mathrm{~d} x \\
& =\sum_{\substack{k, j \in \mathbb{Z} \\
j \neq 0, \mid-j-\leq n}} \int_{\mathbb{R}^{3}}\left(u_{h-j-k}^{n} \cdot \nabla u_{k}^{n}\right) \cdot u_{j}^{n} \mathrm{~d} x \\
& =\sum_{\substack{j \in \mathbb{Z} \\
j \neq 0}} \int_{\mathbb{R}^{3}}\left(\sum_{k \in \mathbb{Z}} u_{h-j-k}^{n} \cdot \nabla u_{k}^{n}\right) \cdot\left(u_{j}^{n} \chi_{h}^{n}(j)\right) \mathrm{d} x
\end{aligned}
$$

with

$$
\chi_{h}^{n}(j):= \begin{cases}1 & \text { if }|h-j| \leq n \\ 0 & \text { if }|h-j|>n\end{cases}
$$


We can, thus, estimate

$$
\begin{aligned}
& \left|\sum_{\substack{|k|,|j| \leq n \\
j \neq 0,|,-j| \leq n}} \int_{\mathbb{R}^{3}}\left(u_{h-j-k}^{n} \cdot \nabla u_{k}^{n}\right) \cdot u_{j}^{n} \mathrm{~d} x-\int_{\mathbb{T}}\left(\int_{\mathbb{R}^{3}} U^{m} \cdot \nabla U^{m} \cdot \mathcal{P}_{\perp} U^{m} \mathrm{~d} x\right) \mathrm{e}^{-i \frac{2 \pi}{T} h t} \mathrm{~d} t\right| \\
& \leq\left|\sum_{\substack{j \in \mathbb{Z} \\
j \neq 0}} \int_{\mathbb{R}^{3}}\left(\sum_{k \in \mathbb{Z}}\left[u_{h-j-k}^{n}-u_{h-j-k}\right] \cdot \nabla u_{k}^{n}\right) \cdot\left(u_{j}^{n} \chi_{h}^{n}(j)\right) \mathrm{d} x\right| \\
& \quad+\left|\sum_{\substack{j \in \mathbb{Z} \\
j \neq 0}} \int_{\mathbb{R}^{3}}\left(\sum_{k \in \mathbb{Z}} u_{h-j-k} \cdot \nabla\left[u_{k}^{n}-u_{k}\right]\right) \cdot\left(u_{j}^{n} \chi_{h}^{n}(j)\right) \mathrm{d} x\right| \\
& \quad+\left|\sum_{\substack{j \in \mathbb{Z} \\
j \neq 0}} \int_{\mathbb{R}^{3}}\left(\sum_{k \in \mathbb{Z}} u_{h-j-k} \cdot \nabla u_{k}\right) \cdot\left(u_{j}^{n} \chi_{h}^{n}(j)-u_{j}\right) \mathrm{d} x\right| \\
& =:
\end{aligned}
$$

In order to estimate $S_{1}$, we utilize the embedding of $\mathrm{W}^{\frac{1}{4}, 2}\left(\mathbb{T} ; Y^{m}\right)$ into $\mathrm{L}^{4}\left(\mathbb{T} ; Y^{m}\right)$ in combination with (45) to conclude that $U_{n}^{m} \rightarrow U^{m}$ in $\mathrm{L}^{4}\left(\mathbb{T}, Y^{m}\right)$ as $n \rightarrow \infty$. Moreover, we recall that

$$
\mathscr{F}_{\mathbb{T}}\left(\left[U_{n}^{m}(\cdot, x)-U^{m}(\cdot, x)\right] \cdot \nabla U_{n}^{m}(\cdot, x)\right)(j)=\sum_{k \in \mathbb{Z}}\left[u_{j-k}^{n}(x)-u_{j-k}(x)\right] \cdot \nabla u_{k}^{n}(x) .
$$

Employing also Plancherel's identity, we deduce

$$
\begin{aligned}
& S_{1} \leq c_{12}\left\|\sum_{k \in \mathbb{Z}}\left[u_{h-j-k}^{n}-u_{h-j-k}\right] \cdot \nabla u_{k}^{n}\right\|_{\ell^{2}\left(Y^{m}\right)}\left\|u_{j}^{n}\right\|_{\ell^{2}\left(Y^{m}\right)} \\
& =c_{13}\left\|\left[U_{n}^{m}-U^{m}\right] \cdot \nabla U_{n}^{m}\right\|_{L^{2}\left(\mathbb{T} ; Y^{m}\right)}\left\|U_{n}^{m}\right\|_{L^{2}\left(\mathbb{T} ; Y^{m}\right)} \\
& \leq c_{14}\left\|U_{n}^{m}-U^{m}\right\|_{L^{4}\left(\mathbb{T} ; Y^{m}\right)}\left\|U_{n}^{m}\right\|_{L^{4}\left(\mathbb{T} ; Y^{m}\right)}\left\|U_{n}^{m}\right\|_{L^{2}\left(\mathbb{T} ; Y^{m}\right)} \quad \rightarrow 0 \quad \text { as } n \rightarrow \infty \text {, }
\end{aligned}
$$

where, in the last inequality, we once more exploit that all norms on $Y^{m}$ are equivalent. In a similar manner, we verify that $S_{2} \rightarrow 0$ as $n \rightarrow \infty$. To estimate $S_{3}$, we proceed as above and obtain

$$
\begin{aligned}
S_{3} \leq & c_{15}\left\|U^{m}\right\|_{L^{4}\left(\mathbb{T} ; Y^{m}\right)}^{2}\left\|u_{j}^{n} \chi_{h}^{n}(j)-u_{j}\right\|_{\ell^{2}\left(Y^{m}\right)} \\
\leq & c_{15}\left\|U^{m}\right\|_{L^{4}\left(\mathbb{T} ; Y^{m}\right)}^{2}\left(\left\|u_{j}^{n}-u_{j}\right\|_{\ell^{2}\left(Y^{m}\right)}+\left\|u_{j}\left(1-\chi_{h}^{n}(j)\right)\right\|_{\ell^{2}\left(Y^{m}\right)}\right) \\
= & c_{16}\left\|U^{m}\right\|_{L^{4}\left(\mathbb{T} ; Y^{m}\right)}^{2}\left(\left\|U_{n}^{m}-U^{m}\right\|_{L^{2}\left(\mathbb{T} ; Y^{m}\right)}+\left\|u_{j}\left(1-\chi_{h}^{n}(j)\right)\right\|_{\ell^{2}\left(Y^{m}\right)}\right) \\
& \rightarrow 0 \text { as } n \rightarrow \infty .
\end{aligned}
$$

We conclude (47). We are now able to pass to the limit $n \rightarrow \infty$ in (42). The limit of the sum on the right-hand side in (42) is computed in (47). Employing (45) to pass to the limit $n \rightarrow \infty$ in the other terms, we conclude that

$$
\begin{aligned}
\int_{\mathbb{T}}( & \left.-i \frac{2 \pi}{\mathcal{T}} h\right)\left(\frac{1}{2} \int_{\mathbb{R}^{3}}\left|\mathcal{P}_{\perp} U^{m}(t, x)\right|^{2} \mathrm{~d} x\right) \mathrm{e}^{-i \frac{2 \pi}{T} h t} \mathrm{~d} t \\
= & -\int_{\mathbb{T}}\left(\int_{\mathbb{R}^{3}} \nabla U^{m}: \nabla \mathcal{P}_{\perp} U^{m} \mathrm{~d} x+\lambda \partial_{1} U^{m} \cdot \mathcal{P}_{\perp} U^{m} \mathrm{~d} x\right) \mathrm{e}^{-i \frac{2 \pi}{T} h t} \mathrm{~d} t \\
& -\int_{\mathbb{T}}\left(\int_{\mathbb{R}^{3}} U^{m} \cdot \nabla U^{m} \cdot \mathcal{P}_{\perp} U^{m} \mathrm{~d} x\right) \mathrm{e}^{-i \frac{2 \pi}{\mathcal{T}} h t} \mathrm{~d} t+\left\langle f_{h}, \mathcal{P}_{\perp} U^{m}\right\rangle .
\end{aligned}
$$


Since

$$
\begin{aligned}
\int_{\mathbb{R}^{3}}\left(U^{m} \cdot \nabla U^{m}\right) \cdot \mathcal{P}_{\perp} U^{m} \mathrm{~d} x \\
\quad=\int_{\mathbb{R}^{3}}\left(\mathcal{P} U^{m} \cdot \nabla \mathcal{P} U^{m}\right) \cdot \mathcal{P}_{\perp} U^{m} \mathrm{~d} x+\int_{\mathbb{R}^{3}}\left(\mathcal{P}_{\perp} U^{m} \cdot \nabla \mathcal{P} U^{m}\right) \cdot \mathcal{P}_{\perp} U^{m} \mathrm{~d} x
\end{aligned}
$$

we obtain from (48) that

$$
\begin{aligned}
\left(i \frac{2 \pi}{\mathcal{T}} h\right) \int_{\mathbb{T}} \frac{1}{2}( & \left.\int_{\mathbb{R}^{3}}\left|\mathcal{P}_{\perp} U^{m}\right|^{2} \mathrm{~d} x\right) \mathrm{e}^{i \frac{2 \pi}{T} h t} \mathrm{~d} t \\
= & \int_{\mathbb{T}} \int_{\mathbb{R}^{3}} \nabla U^{m}: \nabla \mathcal{P}_{\perp} U^{m} \mathrm{~d} x \mathrm{e}^{i \frac{2 \pi}{\mathcal{T}} h t} \mathrm{~d} t \\
& -\lambda \int_{\mathbb{T}} \int_{\mathbb{R}^{3}} \partial_{1} U^{m} \cdot \mathcal{P}_{\perp} U^{m} \mathrm{~d} x \mathrm{e}^{i \frac{2 \pi}{\mathcal{T}} h t} \mathrm{~d} t \\
& +\int_{\mathbb{T}} \int_{\mathbb{R}^{3}}\left(\mathcal{P} U^{m} \cdot \nabla \mathcal{P} U^{m}\right) \cdot \mathcal{P}_{\perp} U^{m} \mathrm{~d} x \mathrm{e}^{i \frac{2 \pi}{T} h t} \mathrm{~d} t \\
& +\int_{\mathbb{T}} \int_{\mathbb{R}^{3}}\left(\mathcal{P}_{\perp} U^{m} \cdot \nabla \mathcal{P} U^{m}\right) \cdot \mathcal{P}_{\perp} U^{m} \mathrm{~d} x \mathrm{e}^{i \frac{2 \pi}{\mathcal{T}} h t} \mathrm{~d} t \\
& -\int_{\mathbb{T}}\left\langle f, \mathcal{P}_{\perp} U^{m}\right\rangle \mathrm{e}^{i \frac{2 \pi}{\mathcal{T}} h t} \mathrm{~d} t
\end{aligned}
$$

for any $h \in \mathbb{Z}$. Now, we put

$$
G(t):=\frac{1}{2} \int_{\mathbb{R}^{3}}\left|\mathcal{P}_{\perp} U^{m}(t, x)\right|^{2} \mathrm{~d} x
$$

and

$$
\begin{aligned}
H(t):= & \int_{\mathbb{R}^{3}} \nabla U^{m}: \nabla \mathcal{P}_{\perp} U^{m} \mathrm{~d} x-\lambda \int_{\mathbb{R}^{3}} \partial_{1} U^{m} \cdot \mathcal{P}_{\perp} U^{m} \mathrm{~d} x+\int_{\mathbb{R}^{3}}\left(\mathcal{P} U^{m} \cdot \nabla \mathcal{P} U^{m}\right) \cdot \mathcal{P}_{\perp} U^{m} \mathrm{~d} x \\
& +\int_{\mathbb{R}^{3}}\left(\mathcal{P}_{\perp} U^{m} \cdot \nabla \mathcal{P} U^{m}\right) \cdot \mathcal{P}_{\perp} U^{m} \mathrm{~d} x-\left\langle f, \mathcal{P}_{\perp} U^{m}\right\rangle .
\end{aligned}
$$

From (46), we already know that $G \in \mathrm{L}^{1}(\mathbb{T})$ with $\|G\|_{\mathrm{L}^{1}(\mathbb{T})} \leq c_{11^{2}}$. From (49), we see that $H$ is the distributional derivative of $G$. We shall verify that $H \in \mathrm{L}^{1}(\mathbb{T})$ and estimate $\|H\|_{\mathrm{L}^{1}(\mathbb{T})}$. For this purpose, we estimate, recalling (46),

$$
\int_{\mathbb{T}}\left|\int_{\mathbb{R}^{3}} \nabla U^{m}: \nabla \mathcal{P}_{\perp} U^{m} \mathrm{~d} x\right| \mathrm{d} t \leq\left\|U^{m}\right\|_{\mathrm{L}^{2}\left(\mathbb{T} ; \mathrm{D}_{0, \sigma}^{1,2}\left(\mathbb{R}^{3}\right)\right)}^{2} \leq c_{11}^{2}
$$

and

$$
\int_{\mathbb{T}}\left|\int_{\mathbb{R}^{3}} \partial_{1} U^{m} \cdot \mathcal{P}_{\perp} U^{m} \mathrm{~d} x\right| \mathrm{d} t \leq\left\|U^{m}\right\|_{\mathrm{L}^{2}\left(\mathbb{T} ; \mathrm{D}_{0, \sigma}^{1,2}\left(\mathbb{R}^{3}\right)\right)}\left\|\mathcal{P}_{\perp} U^{m}\right\|_{\mathrm{L}^{2}\left(\mathbb{T} ; \mathrm{L}^{2}\left(\mathbb{R}^{3}\right)\right)} \leq c_{11}{ }^{2} .
$$

Employing Lemma 1, (6), and Hölder's inequality, we find, recalling again (46), that

$$
\begin{aligned}
& \int_{\mathbb{T}}\left|\int_{\mathbb{R}^{3}}\left(\mathcal{P} U^{m} \cdot \nabla \mathcal{P} U^{m}\right) \cdot \mathcal{P}_{\perp} U^{m} \mathrm{~d} x\right| \mathrm{d} t \\
& \leq \int_{\mathbb{T}}\left\|\mathcal{P} U^{m}\right\|_{6}\left\|\nabla \mathcal{P} U^{m}\right\|_{2}\left\|\mathcal{P}_{\perp} U^{m}(t)\right\|_{3} \mathrm{~d} t \\
& \leq\left\|\nabla \mathcal{P} U^{m}\right\|_{2}^{2} \int_{\mathbb{T}}\left\|\nabla \mathcal{P}_{\perp} U^{m}(t)\right\|_{2}^{\frac{1}{2}}\left\|\mathcal{P}_{\perp} U^{m}(t)\right\|_{2}^{\frac{1}{2}} \mathrm{~d} t \\
&\left.\left.\leq\left\|\nabla \mathcal{P} U^{m}\right\|_{2}^{2}\left\|\mathcal{P}_{\perp} U^{m}\right\|_{\mathrm{L}^{2}\left(\mathbb{T} ; \mathrm{D}_{0, \sigma}^{1,2}\right.}^{\frac{1}{2}} \mathbb{R}^{3}\right)\right) \\
& \leq \mathcal{P}_{\perp} U^{m} \|_{\mathrm{L}^{2}\left(\mathbb{T} ; \mathrm{L}^{2}\left(\mathbb{R}^{3}\right)\right)}^{\frac{1}{2}} \\
& \leq c_{11}{ }^{3} .
\end{aligned}
$$


Similarly, we estimate

$$
\begin{aligned}
\int_{\mathbb{T}}\left|\int_{\mathbb{R}^{3}}\left(\mathcal{P}_{\perp} U^{m} \cdot \nabla \mathcal{P} U^{m}\right) \cdot \mathcal{P}_{\perp} U^{m} \mathrm{~d} x\right| \mathrm{d} t \\
\leq\left\|\nabla \mathcal{P} U^{m}\right\|_{2} \int_{\mathbb{T}}\left\|\mathcal{P}_{\perp} U^{m}(t)\right\|_{4}^{2} \mathrm{~d} t \\
\leq\left\|\nabla \mathcal{P} U^{m}\right\|_{2} \int_{\mathbb{T}}\left\|\mathcal{P}_{\perp} U^{m}(t)\right\|_{2}^{\frac{1}{2}}\left\|\nabla \mathcal{P}_{\perp} U^{m}(t)\right\|_{2}^{\frac{3}{2}} \mathrm{~d} t \\
\leq\left\|\nabla \mathcal{P} U^{m}\right\|_{2}\left\|\mathcal{P}_{\perp} U^{m}\right\|_{\mathrm{L}^{2}\left(\mathbb{T} ; \mathrm{L}^{2}\left(\mathbb{R}^{3}\right)\right)}^{\frac{1}{2}}\left\|\mathcal{P}_{\perp} U^{m}\right\|_{\mathrm{L}^{2}\left(\mathbb{T} ; \mathrm{D}_{0, \sigma}^{1,2}\left(\mathbb{R}^{3}\right)\right)}^{\frac{3}{2}} \\
\leq c_{11}{ }^{3} .
\end{aligned}
$$

Finally, we observe that

$$
\begin{aligned}
\int_{\mathbb{T}}\left|\left\langle f, \mathcal{P}_{\perp} U^{m}\right\rangle\right| \mathrm{d} t & \leq\|f\|_{\mathrm{L}^{2}\left(\mathbb{T} ; \mathrm{D}_{0}^{-1,2}\left(\mathbb{R}^{3}\right)\right)}\left\|\mathcal{P}_{\perp} U^{m}\right\|_{\mathrm{L}^{2}\left(\mathbb{T} ; \mathrm{D}_{0, \sigma}^{1,2}\left(\mathbb{R}^{3}\right)\right)} \\
& \leq c_{11}\|f\|_{\mathrm{L}^{2}\left(\mathbb{T} ; \mathrm{D}_{0}^{-1,2}\left(\mathbb{R}^{3}\right)\right)} .
\end{aligned}
$$

By (50)-(54), it follows that $H \in \mathrm{L}^{1}(\mathbb{T})$. We, thus, deduce $G \in \mathrm{W}^{1,1}(\mathbb{T})$ with

$$
\|G\|_{\mathrm{W}^{1,1}(\mathbb{T})} \leq c_{11}^{2}+\lambda c_{11}^{2}+c_{11}^{3}+c_{11}\|f\|_{\mathrm{L}^{2}\left(\mathbb{T} ; \mathrm{D}_{0}^{-1,2}\left(\mathbb{R}^{3}\right)\right)} .
$$

Since $W^{1,1}(\mathbb{T})$ is continuously embedded in $L^{\infty}(\mathbb{T})$, we conclude that

$$
\left\|\mathcal{P}_{\perp} U^{m}\right\|_{L^{\infty}\left(\mathbb{T} ; L^{2}\left(\mathbb{R}^{3}\right)\right)}=4\|G\|_{L^{\infty}(\mathbb{T})}^{\frac{1}{2}} \leq c_{17}
$$

with $c_{17}=c_{17}(\lambda,\|f\|)$ independent on $m$.

We now verify that $U^{m}$ is an approximate solution to (1). For any $\Phi \in X_{n_{0}}^{m}$, we have

$$
\int_{\mathbb{T}} \int_{\mathbb{R}^{3}}-U_{n}^{m} \cdot \partial_{t} \Phi+\nabla U_{n}^{m}: \nabla \Phi-\lambda \partial_{1} U_{n}^{m} \cdot \Phi+\left(U_{n}^{m} \cdot \nabla U_{n}^{m}\right) \cdot \Phi \mathrm{d} x \mathrm{~d} t=\int_{\mathbb{T}}\langle f, \Phi\rangle \mathrm{d} t
$$

for $n \geq n_{0}$. We can pass to the limit $n \rightarrow \infty$ in (56) in the same manner as we derived (49) by passing to the limit $n \rightarrow \infty$ in (42). In particular, we can utilize (45) to show

$$
\begin{aligned}
\forall \Phi \in \bigcup_{n_{0} \in \mathbb{N}} X_{n_{0}}^{m}: \quad & \int_{\mathbb{T}} \int_{\mathbb{R}^{3}}-U^{m} \cdot \partial_{t} \Phi+\nabla U^{m}: \nabla \Phi-\lambda \partial_{1} U^{m} \cdot \Phi+\left(U^{m} \cdot \nabla U^{m}\right) \cdot \Phi \mathrm{d} x \mathrm{~d} t \\
& =\int_{\mathbb{T}}\langle f, \Phi\rangle \mathrm{d} t .
\end{aligned}
$$

We summarize at this point that $U^{m} \in \mathrm{L}^{2}\left(\mathbb{T} ; \mathrm{D}_{0, \sigma}^{1,2}\left(\mathbb{R}^{3}\right)\right)$ is an approximate solution to (1) that satisfies (46) and (55).

To obtain a proper weak solution to (1), we let $m \rightarrow \infty$. By (46), the sequence $\left\{U^{m}\right\}_{m=1}^{\infty}$ is bounded in $\mathrm{L}^{2}\left(\mathbb{T} ; \mathrm{D}_{0, \sigma}^{1,2}\left(\mathbb{R}^{3}\right)\right)$. Thus, there is a $U \in \mathrm{L}^{2}\left(\mathbb{T} ; \mathrm{D}_{0, \sigma}^{1,2}\left(\mathbb{R}^{3}\right)\right)$ and a subsequence of $\left\{U^{m}\right\}_{m=1}^{\infty}$, which, for simplicity, we still denote by $\left\{U^{m}\right\}_{m=1}^{\infty}$, which converges weakly towards $U$ as $m \rightarrow \infty$. In view of (46) and (55), we see that

$$
\gamma \in\left[0, \frac{1}{3}\right): \quad \mathcal{P}_{\perp} U \in \mathrm{W}^{\gamma, 2}\left(\mathbb{T} ; \mathrm{L}^{2}\left(\mathbb{R}^{3}\right)^{3}\right) \cap \mathrm{L}^{\infty}\left(\mathbb{T} ; \mathrm{L}^{2}\left(\mathbb{R}^{3}\right)^{3}\right),
$$


and we can pick any $\gamma \in\left(0, \frac{1}{3}\right)$ and choose the subsequence in such a way that

$$
\begin{array}{ll}
U^{m} \rightarrow U & \text { in } \mathrm{L}^{2}\left(\mathbb{T} ; \mathrm{D}_{0, \sigma}^{1,2}\left(\mathbb{R}^{3}\right)\right), \\
\mathcal{P}_{\perp} U^{m} \rightarrow \mathcal{P}_{\perp} U & \text { in } \mathrm{W}^{\gamma, 2}\left(\mathbb{T} ; \mathrm{L}^{2}\left(\mathbb{R}^{3}\right)^{3}\right), \\
\mathcal{P}_{\perp} U^{m} \rightarrow^{*} \mathcal{P}_{\perp} U & \text { in } \mathrm{L}^{\infty}\left(\mathbb{T} ; \mathrm{L}^{2}\left(\mathbb{R}^{3}\right)^{3}\right) \quad \text { as } m \rightarrow \infty,
\end{array}
$$

where $\rightarrow^{*}$ denotes convergence with respect to the weak-topology of $\mathrm{L}^{\infty}\left(\mathbb{T} ; \mathrm{L}^{2}\left(\mathbb{R}^{3}\right)^{3}\right)$. For $K \subset \mathbb{R}^{3}$ a compact set, $\mathrm{W}^{\gamma, 2}\left(\mathbb{T} ; \mathrm{L}^{2}\left(\mathbb{R}^{3}\right)^{3}\right) \cap \mathrm{L}^{2}\left(\mathbb{T} ; \mathrm{D}_{0}^{1,2}\left(\mathbb{R}^{3}\right)^{3}\right)$ is compactly embedded in $\mathrm{L}^{2}\left(\mathbb{T} ; \mathrm{L}^{2}(K)^{3}\right)$; see for example [14] (Chapter II, $\$ 2$, Theorem 2.2). Moreover, $\mathrm{D}_{0, \sigma}^{1,2}\left(\mathbb{R}^{3}\right)$ is compactly embedded in $\mathrm{L}^{2}(K)^{3}$. We, therefore, also have

$$
\begin{array}{lll}
\forall K \subset \mathbb{R}^{3}, K \text { compact : } & \mathcal{P}_{\perp} U^{m} \rightarrow \mathcal{P}_{\perp} U & \text { in } \mathrm{L}^{2}\left(\mathbb{T} ; \mathrm{L}^{2}(K)^{3}\right), \\
\forall K \subset \mathbb{R}^{3}, K \text { compact : } & \mathcal{P} U^{m} \rightarrow \mathcal{P} U & \text { in } \mathrm{L}^{2}(K)^{3} .
\end{array}
$$

By virtue of (57) and (59)-(60), it can now be shown that $U$ is a weak solution to (1). Consider first $\Phi \in X_{n_{0}}^{m_{0}}$ for some $n_{0}, m_{0} \in \mathbb{N}$. With this test function fixed, we pass to the limit $m \rightarrow \infty$ in (57). It is easy to pass to the limit in the terms that are linear in $U^{m}$. To verify that we can also pass to the limit in the nonlinear term, we first compute

$$
\begin{aligned}
\int_{\mathbb{T}} \int_{\mathbb{R}^{3}}\left(U^{m} \cdot \nabla U^{m}\right) \cdot \Phi \mathrm{d} x \mathrm{~d} t= & \int_{\mathbb{T}} \int_{\mathbb{R}^{3}}\left(\mathcal{P} U^{m} \cdot \nabla \Phi\right) \cdot \mathcal{P} U^{m} \mathrm{~d} x \mathrm{~d} t \\
& +\int_{\mathbb{T}} \int_{\mathbb{R}^{3}}\left(\mathcal{P} U^{m} \cdot \nabla \Phi\right) \cdot \mathcal{P}_{\perp} U^{m} \mathrm{~d} x \mathrm{~d} t \\
& +\int_{\mathbb{T}} \int_{\mathbb{R}^{3}}\left(\mathcal{P}_{\perp} U^{m} \cdot \nabla \Phi\right) \cdot \mathcal{P} U^{m} \mathrm{~d} x \mathrm{~d} t \\
& +\int_{\mathbb{T}} \int_{\mathbb{R}^{3}}\left(\mathcal{P}_{\perp} U^{m} \cdot \nabla \Phi\right) \cdot \mathcal{P}_{\perp} U^{m} \mathrm{~d} x \mathrm{~d} t .
\end{aligned}
$$

We can estimate

$$
\begin{aligned}
\left|\int_{\mathbb{T}} \int_{\mathbb{R}^{3}}\left(\mathcal{P} U^{m} \cdot \nabla \Phi\right) \cdot \mathcal{P} U^{m} \mathrm{~d} x \mathrm{~d} t-\int_{\mathbb{T}} \int_{\mathbb{R}^{3}}(\mathcal{P} U \cdot \nabla \Phi) \cdot \mathcal{P} U \mathrm{~d} x \mathrm{~d} t\right| \\
\leq c_{18}\left\|\mathcal{P} U^{m}-\mathcal{P} U\right\|_{\mathrm{L}^{2}(K)}\left(\|\mathcal{P} U\|_{\mathrm{L}^{2}(K)}+\left\|\mathcal{P} U^{m}\right\|_{\mathrm{L}^{2}(K)}\right)
\end{aligned}
$$

with $K:=\bigcup_{j \leq m_{0}}$ supp $\psi_{j}$ and $c_{18}$ independent on $m$. Similarly, we estimate

$$
\begin{gathered}
\left|\int_{\mathbb{T}} \int_{\mathbb{R}^{3}}\left(\mathcal{P} U^{m} \cdot \nabla \Phi\right) \cdot \mathcal{P}_{\perp} U^{m} \mathrm{~d} x \mathrm{~d} t-\int_{\mathbb{T}} \int_{\mathbb{R}^{3}}(\mathcal{P} U \cdot \nabla \Phi) \cdot \mathcal{P}_{\perp} U \mathrm{~d} x \mathrm{~d} t\right| \\
\leq c_{19}\left(\left\|\mathcal{P} U^{m}-\mathcal{P} U\right\|_{\mathrm{L}^{2}(K)}\left\|\mathcal{P}_{\perp} U^{m}\right\|_{\mathrm{L}^{2}\left(\mathbb{T} ; \mathrm{L}^{2}(K)\right)}\right. \\
\left.+\|\mathcal{P} U\|_{\mathrm{L}^{2}(K)}\left\|\mathcal{P}_{\perp} U^{m}-\mathcal{P}_{\perp} U\right\|_{\mathrm{L}^{2}\left(\mathbb{T} ; \mathrm{L}^{2}(K)\right)}\right),
\end{gathered}
$$

and

$$
\begin{gathered}
\left|\int_{\mathbb{T}} \int_{\mathbb{R}^{3}}\left(\mathcal{P}_{\perp} U^{m} \cdot \nabla \Phi\right) \cdot \mathcal{P} U^{m} \mathrm{~d} x \mathrm{~d} t-\int_{\mathbb{T}} \int_{\mathbb{R}^{3}}\left(\mathcal{P}_{\perp} U \cdot \nabla \Phi\right) \cdot \mathcal{P} U \mathrm{~d} x \mathrm{~d} t\right| \\
\leq c_{20}\left(\left\|\mathcal{P} U^{m}-\mathcal{P} U\right\|_{\mathrm{L}^{2}(K)}\left\|\mathcal{P}_{\perp} U^{m}\right\|_{\mathrm{L}^{2}\left(\mathbb{T} ; \mathrm{L}^{2}(K)\right)}\right. \\
\left.\quad+\|\mathcal{P} U\|_{\mathrm{L}^{2}(K)}\left\|\mathcal{P}_{\perp} U^{m}-\mathcal{P}_{\perp} U\right\|_{\mathrm{L}^{2}\left(\mathbb{T} ; \mathrm{L}^{2}(K)\right)}\right) .
\end{gathered}
$$


Finally, we also have

$$
\begin{aligned}
\left|\int_{\mathbb{T}} \int_{\mathbb{R}^{3}}\left(\mathcal{P}_{\perp} U^{m} \cdot \nabla \Phi\right) \cdot \mathcal{P}_{\perp} U^{m} \mathrm{~d} x \mathrm{~d} t-\int_{\mathbb{T}} \int_{\mathbb{R}^{3}}\left(\mathcal{P}_{\perp} U \cdot \nabla \Phi\right) \cdot \mathcal{P}_{\perp} U \mathrm{~d} x \mathrm{~d} t\right| \\
\leq c_{21}\left\|\mathcal{P}_{\perp} U^{m}-\mathcal{P} U\right\|_{\mathrm{L}^{2}\left(\mathbb{T} ; \mathrm{L}^{2}(K)\right)}\left(\left\|\mathcal{P}_{\perp} U\right\|_{\mathrm{L}^{2}\left(\mathbb{T} ; \mathrm{L}^{2}(K)\right)}+\left\|\mathcal{P}_{\perp} U^{m}\right\|_{\mathrm{L}^{2}\left(\mathbb{T} ; \mathrm{L}^{2}(K)\right)}\right) .
\end{aligned}
$$

Clearly, $K \subset \mathbb{R}^{3}$ is compact. Thus, the estimates above together with (60) imply that we can pass to the limit $m \rightarrow \infty$ in (61) to obtain

$$
\lim _{m \rightarrow \infty} \int_{\mathbb{T}} \int_{\mathbb{R}^{3}}\left(U^{m} \cdot \nabla U^{m}\right) \cdot \Phi \mathrm{d} x \mathrm{~d} t=\int_{\mathbb{T}} \int_{\mathbb{R}^{3}}(U \cdot \nabla U) \cdot \Phi \mathrm{d} x \mathrm{~d} t .
$$

Returning to (57), we find, after letting $m \rightarrow \infty$, that

$$
\begin{aligned}
& \forall \Phi \in \bigcup_{n_{0}, m_{0} \in \mathbb{N}} X_{n_{0}}^{m_{0}}: \\
& \quad \int_{\mathbb{T}} \int_{\mathbb{R}^{3}}-U \cdot \partial_{t} \Phi+\nabla U: \nabla \Phi-\lambda \partial_{1} U \cdot \Phi+(U \cdot \nabla U) \cdot \Phi \mathrm{d} x \mathrm{~d} t=\int_{\mathbb{T}}\langle f, \Phi\rangle \mathrm{d} t .
\end{aligned}
$$

To finalize the proof, we need to extend the identity above to all $\Phi \in C_{0, \sigma}^{\infty}\left(\mathbb{T} \times \mathbb{R}^{3}\right)$. For this purpose, we put

$$
\Phi_{j, k}(t, x):=\left(1+\left(\frac{2 \pi}{\mathcal{T}}\right)^{2}|k|^{2}\right)^{-\frac{1}{2}} \mathrm{e}^{i \frac{2 \pi}{T} k t} \psi_{j}(x)
$$

and utilize that $\left\{\Phi_{j, k} \mid k, j \in \mathbb{Z}\right\}$ is an orthonormal basis in $\mathrm{W}^{1,2}\left(\mathbb{T} ; \mathrm{W}_{0, \sigma}^{1,2}\left(\mathbb{R}^{3}\right)\right)$. Consider $\Phi \in C_{0, \sigma}^{\infty}\left(\mathbb{T} \times \mathbb{R}^{3}\right)$. Let $\langle\cdot, \cdot\rangle_{\mathrm{W}^{1,2}\left(\mathbb{T} ; \mathrm{W}^{1,2}\left(\mathbb{R}^{3}\right)^{3}\right)}$ denote the canonical inner product of $\mathrm{W}^{1,2}\left(\mathbb{T} ; \mathrm{W}^{1,2}\left(\mathbb{R}^{3}\right)^{3}\right)$ and define

$$
\Phi_{m}(t, x):=\sum_{|k|,|j| \leq m}\left\langle\Phi, \Phi_{j, k}\right\rangle_{\mathrm{W}^{1,2}\left(\mathbb{T} ; \mathrm{W}^{1,2}\left(\mathbb{R}^{3}\right)^{3}\right)} \Phi_{j, k} .
$$

We, then, use $\Phi_{m}$ as a test function in (62) and pass to the limit $m \rightarrow \infty$. Observe that

$$
\begin{aligned}
\left|\int_{\mathbb{T}} \int_{\mathbb{R}^{3}} U \cdot \partial_{t} \Phi-U \cdot \partial_{t} \Phi_{m} \mathrm{~d} x \mathrm{~d} t\right| & =\left|\int_{\mathbb{T}} \int_{\mathbb{R}^{3}} \mathcal{P}_{\perp} U \cdot \partial_{t} \Phi-\mathcal{P}_{\perp} U \cdot \partial_{t} \Phi_{m} \mathrm{~d} x \mathrm{~d} t\right| \\
& \leq\left\|\mathcal{P}_{\perp} U\right\|_{\mathrm{L}^{2}\left(\mathbb{T} ; \mathrm{L}^{2}\left(\mathbb{R}^{3}\right)\right)}\left\|\Phi-\Phi_{m}\right\|_{\mathrm{W}^{1,2}\left(\mathrm{~L}^{2}\left(\mathbb{R}^{3}\right)\right)}
\end{aligned}
$$

whence

$$
\lim _{m \rightarrow \infty} \int_{\mathbb{T}} \int_{\mathbb{R}^{3}} U \cdot \partial_{t} \Phi_{m} \mathrm{~d} x \mathrm{~d} t=\int_{\mathbb{T}} \int_{\mathbb{R}^{3}} U \cdot \partial_{t} \Phi \mathrm{d} x \mathrm{~d} t .
$$

We also note that

$$
\begin{aligned}
\left|\int_{\mathbb{T}} \int_{\mathbb{R}^{3}}(U \cdot \nabla U) \cdot \Phi-(U \cdot \nabla U) \cdot \Phi_{m} \mathrm{~d} x \mathrm{~d} t\right| \\
\leq \int_{\mathbb{T}}\|U(t)\|_{6}\|\nabla U(t)\|_{2}\left\|\Phi(t)-\Phi_{m}(t)\right\|_{3} \mathrm{~d} t \\
\leq c_{22}\|U\|_{\mathrm{L}^{2}\left(\mathbb{T} ; \mathrm{D}_{0, \sigma}^{1,2}\left(\mathbb{R}^{3}\right)\right)}^{2} \sup _{t \in \mathrm{T}}\left\|\Phi-\Phi_{m}\right\|_{\mathrm{W}_{0, \sigma}^{1,2}\left(\mathbb{R}^{3}\right)} \\
\leq c_{23}\|U\|_{\mathrm{L}^{2}\left(\mathbb{T} ; \mathrm{D}_{0, \sigma}^{1,2}\left(\mathbb{R}^{3}\right)\right)}\left\|\Phi-\Phi_{m}\right\|_{\mathrm{W}^{1,2}\left(\mathrm{~W}_{0, \sigma}^{1,2}\left(\mathbb{R}^{3}\right)\right)^{\prime}}
\end{aligned}
$$

which implies

$$
\lim _{m \rightarrow \infty} \int_{\mathbb{T}} \int_{\mathbb{R}^{3}}(U \cdot \nabla U) \cdot \Phi_{m} \mathrm{~d} x \mathrm{~d} t=\int_{\mathbb{T}} \int_{\mathbb{R}^{3}}(U \cdot \nabla U) \cdot \Phi \mathrm{d} x \mathrm{~d} t .
$$


It is now easy to verify, after inserting $\Phi_{m}$ as a test function in (62) and passing to the limit $m \rightarrow \infty$, that $U$ and $\Phi$ satisfy (13). Thus, recalling (58), we have shown that $U \in$ $\mathrm{L}^{2}\left(\mathbb{T} ; \mathrm{D}_{0, \sigma}^{1,2}\left(\mathbb{R}^{3}\right)\right)$ is a weak time-periodic solution to (1) that satisfies (14)-(16).

Remark 3. Observe that the condition $\gamma>\frac{1}{3}$ is needed to conclude that (33) is not dictated by the regularity of the data $f$. In other words, assuming more regularity on the data $f$ will not lead to any improvement in (16) and, thereby, to better regularity of the solution $u$, at least not in the proof above.

Proof of Theorem 1. As noted in Section 2.3, the quotient mapping $\Pi: \mathbb{R} \rightarrow \mathbb{T}$, employed as a pull-back operator, yields a bijection between $\mathcal{T}$-time-periodic functions defined on $\mathbb{R}$ and functions defined on the torus $\mathbb{T}$. Employing this bijection, and recalling (6), one readily verifies that Theorem 1 follows from Theorem 2 .

Remark 4. We shall briefly compare Theorem 2 with the similar well-known result for the the initial-value Navier-Stokes problem

$$
\begin{cases}\partial_{t} u-\Delta u-\lambda \partial_{1} u+\nabla \mathfrak{p}+u \cdot \nabla u=f & \text { in }(0, T) \times \mathbb{R}^{3}, \\ \operatorname{div} u=0 & \text { in }(0, T) \times \mathbb{R}^{3}, \\ u(0, \cdot)=u_{0} & \text { in } \mathbb{R}^{3} .\end{cases}
$$

The existence of a weak solution $u \in \mathrm{L}^{2}\left(0, T ; \mathrm{D}_{0, \sigma}^{1,2}\left(\mathbb{R}^{3}\right)\right)$ to (63) was originally shown by Leray in [2]. A proof based on a Galerkin approximation was given by Hopf in [1]. Compared to the proof of Theorem 2 above, which is also based on a Galerkin approximation, it is much simpler in the proof of Hopf to obtain $u \in \mathrm{L}^{\infty}\left(0, T ; \mathrm{L}^{2}\left(\mathbb{R}^{3}\right)^{3}\right)$. Indeed, multiplying (63) $)_{1}$ with $u$ and performing for arbitrary $t \in(0, t)$ the formal integration

$$
\int_{0}^{t} \int_{\mathbb{R}^{3}} \partial_{t} u \cdot u-\Delta u \cdot u-\lambda \partial_{1} u \cdot u+\nabla \mathfrak{p} \cdot u+(u \cdot \nabla u) \cdot u \mathrm{~d} x \mathrm{~d} t=\int_{0}^{t} \int_{\mathbb{R}^{3}} f \cdot u \mathrm{~d} x \mathrm{~d} t,
$$

one finds, after a formal integration by parts and a simple estimate, that

$$
\|u(t)\|_{2} \leq\|u(0)\|_{2}+2\|f\|_{\mathrm{L}^{2}\left(0, T ; \mathrm{D}_{0}^{-1,2}\left(\mathbb{R}^{3}\right)\right)}\|u\|_{\mathrm{L}^{2}\left(0, T ; \mathrm{D}_{0, \sigma}^{1,2}\left(\mathbb{R}^{3}\right)\right)} .
$$

The computation above can only be performed formally at first; however, since it is valid on the finite-dimensional level in the Galerkin approximation, it eventually leads to the validity of (64) also for the final weak solution $u$. Since $\|u(0)\|_{2}$ equals the prescribed quantity $u_{0}$, (64) implies that $\|u(t)\|_{2}$ is bounded independently on $t$ and, thus, $u \in \mathrm{L}^{\infty}\left(0, T ; \mathrm{L}^{2}\left(\mathbb{R}^{3}\right)^{3}\right)$. In contrast, in the time-periodic case, there is no information available on $\|u(0)\|_{2}$. Consequently, the simple argument that yields $u \in \mathrm{L}^{\infty}\left(0, T ; \mathrm{L}^{2}\left(\mathbb{R}^{3}\right)^{3}\right)$ for the initial-value problem cannot be used in the time-periodic case.

Remark 5. It may seem surprising at first that Theorem 2 only establishes that the projection $\mathcal{P}_{\perp} u$ lies in $\mathrm{L}^{\infty}\left(0, \mathcal{T} ; \mathrm{L}^{2}\left(\mathbb{R}^{3}\right)^{3}\right)$ and not $u$ itself, which, as shown above, can be proved rather easily for the corresponding initial-value problem. A short investigation, however, reveals that $u$ cannot in general belong to $\mathrm{L}^{\infty}\left(0, \mathcal{T} ; \mathrm{L}^{2}\left(\mathbb{R}^{3}\right)^{3}\right)$ in the time-periodic case. If, for example, the prescribed data $f$ is time independent, the Galerkin method employed in the proof of Theorem 2 may yield a time-independent weak solution $u$, that is, a steady-state solution to

$$
\begin{cases}-\Delta u-\lambda \partial_{1} u+\nabla \mathfrak{p}+u \cdot \nabla u=f & \text { in } \mathbb{R}^{3}, \\ \operatorname{div} u=0 & \text { in } \mathbb{R}^{3} .\end{cases}
$$

Indeed, it is easy to verify that the vector $\xi_{0}=\left(\xi^{\#}, \xi^{*}, \xi^{0}\right) \in \mathbb{R}^{m \times n} \times \mathbb{R}^{m \times n} \times \mathbb{R}^{m}$ in (19) may be chosen such that $\xi^{\#}=\xi^{*}=0$, if $f$ is time independent. By explicitly choosing $\xi_{0}$ in this 
way, the existence part of the proof reduces completely to the proof of [13] (Theorem IX.4.1), which yields a weak so-called Leray solution to (65). Provided $f$ is of compact support and possesses a certain amount of regularity, it was shown by Finn [15], in the case $\lambda \neq 0$, that such a solution does not belong to $\mathrm{L}^{2}\left(\mathbb{R}^{3}\right)^{3}$ when $\int_{\mathbb{R}^{3}} f \mathrm{~d} x \neq 0$; see also [13] (Theorem IX.7.2). Consequently, $u \notin \mathrm{L}^{\infty}\left(0, T ; \mathrm{L}^{2}\left(\mathbb{R}^{3}\right)^{3}\right)$ in this case. If we further assume that $f$ is sufficiently small, we can use result by Korolev and Šverák [16] to derive $u \notin \mathrm{L}^{\infty}\left(0, T ; \mathrm{L}^{2}\left(\mathbb{R}^{3}\right)^{3}\right)$ also in the case $\lambda=0$.

\section{Regularity Properties}

The purpose of this section is to show that the weak solution from Theorem 2 is continuous in time with respect an appropriate topology. Continuity in time is needed to conclude that the solution is in fact time-periodic in a classical pointwise fashion. It is easy to verify that a completely analogous statement follows for the solution in Theorem 1.

From the weak formulation (13), time regularity of the solution in a relatively weak topology can be derived almost directly. More specifically, we have:

Lemma 2. Let $f \in \mathrm{L}^{2}\left(\mathbb{T} ; \mathrm{D}_{0}^{-1,2}\left(\mathbb{R}^{3}\right)^{3}\right)$ and $\lambda \in \mathbb{R}$. The weak time-periodic solution $u \in$ $\mathrm{L}^{2}\left(\mathbb{T} ; \mathrm{D}_{0, \sigma}^{1,2}\left(\mathbb{R}^{3}\right)\right)$ from Theorem 2 satisfies

$$
\forall N \in \mathbb{N}: \quad u \in \mathrm{W}^{1,1}\left(\mathbb{T} ; \mathrm{D}_{0, \sigma}^{-1,2}\left(B_{N}\right)\right)
$$

with

$$
\begin{aligned}
& \forall \varphi \in \mathrm{D}_{0, \sigma}^{1,2}\left(B_{N}\right): \\
& -\left\langle\partial_{t} u(t), \varphi\right\rangle=\int_{\mathbb{R}^{3}} \nabla u(t): \nabla \varphi-\lambda \partial_{1} u(t) \cdot \varphi+(u(t) \cdot \nabla u(t)) \cdot \varphi-\langle f(t), \varphi\rangle \mathrm{d} x
\end{aligned}
$$

for a.e. $t \in \mathbb{T}$.

Proof. Let $N \in \mathbb{N}$. For $t \in \mathbb{T}$, we let $g(t) \in \mathrm{D}_{0, \sigma}^{-1,2}\left(B_{N}\right)$ denote the functional

$$
\langle g(t), \varphi\rangle:=\int_{\mathbb{R}^{3}} \nabla u(t): \nabla \varphi-\lambda \partial_{1} u(t) \cdot \varphi+(u(t) \cdot \nabla u(t)) \cdot \varphi \mathrm{d} x-\langle f(t), \varphi\rangle .
$$

We can estimate

$$
\begin{aligned}
& |\langle g(t), \varphi\rangle| \\
& \quad \leq\|\nabla u(t)\|_{2}\|\nabla \varphi\|_{2}+\lambda\|\nabla u(t)\|_{2}\|\varphi\|_{2}+\left|\int_{\mathbb{R}^{3}}(u(t) \cdot \nabla \varphi) \cdot u(t) \mathrm{d} x\right|+|f(t)|_{-1,2}\|\nabla \varphi\|_{2} \\
& \quad \leq c_{1}\left(\|\nabla u(t)\|_{2}+\|u(t)\|_{\mathrm{L}^{4}\left(B_{N}\right)}^{2}+|f(t)|_{-1,2}\right)\|\nabla \varphi\|_{2} \\
& \quad \leq c_{2}\left(\|\nabla u(t)\|_{2}+\|\nabla u(t)\|_{2}^{2}+|f(t)|_{-1,2}\right)\|\nabla \varphi\|_{2},
\end{aligned}
$$

with $c_{2}=c_{2}(N)$. It follows that $g \in \mathrm{L}^{1}\left(\mathbb{T} ; \mathrm{D}_{0, \sigma}^{-1,2}\left(B_{N}\right)\right)$. Since $u \in \mathrm{L}^{2}\left(\mathbb{T} ; \mathrm{D}_{0, \sigma}^{1,2}\left(\mathbb{R}^{3}\right)\right)$, we immediately obtain $u \in \mathrm{L}^{1}\left(\mathbb{T} ; \mathrm{D}_{0, \sigma}^{-1,2}\left(B_{N}\right)\right)$. To show (66) and (67), we, therefore, only need to verify $-\partial_{t} u=g$. For this purpose, we want to use, for arbitrary $k \in \mathbb{Z}$ and $\varphi \in \mathrm{D}_{0, \sigma}^{1,2}\left(B_{N}\right), \Phi(t, x):=\varphi(x) \mathrm{e}^{i \frac{2 \pi}{\mathcal{T}} k t}$ as a test function in the weak formulation (13). Since $\Phi(t, x)$ is not admissible as a test function, we first approximate $\varphi \in \mathrm{D}_{0, \sigma}^{1,2}\left(B_{N}\right)$ by a sequence $\left\{\varphi_{n}\right\}_{n=1}^{\infty} \subset C_{0, \sigma}^{\infty}\left(B_{N}\right)$ in the $|\cdot|_{1,2}$-norm. Using $\Phi_{n}(t, x):=\varphi_{n}(x) \mathrm{e}^{i \frac{2 \pi}{\mathcal{T}} k t}$ as a test function and subsequently letting $n \rightarrow \infty$, it is easy to verify that

$$
\begin{aligned}
i \frac{2 \pi}{\mathcal{T}} k \int_{\mathbb{T}} & \int_{\mathbb{R}^{3}} u(t, x) \cdot \varphi(x) \mathrm{d} x \mathrm{e}^{i \frac{2 \pi}{\mathcal{T}} k t} \mathrm{~d} t \\
& =\int_{\mathbb{T}}\left(\int_{\mathbb{R}^{3}} \nabla u: \nabla \varphi-\lambda \partial_{1} u \cdot \varphi+(u \cdot \nabla u) \cdot \varphi \mathrm{d} x\right) \mathrm{e}^{i \frac{2 \pi}{\mathcal{T}} k t}-\langle f, \varphi\rangle \mathrm{e}^{i \frac{2 \pi}{\mathcal{T}} k t} \mathrm{~d} t .
\end{aligned}
$$


We deduce that

$$
i \frac{2 \pi}{\mathcal{T}} k \int_{\mathbb{T}} u(t) \mathrm{e}^{i \frac{2 \pi}{\mathcal{T}} k t} \mathrm{~d} t=\int_{\mathbb{T}} g(t) \mathrm{e}^{i \frac{2 \pi}{\mathcal{T}} k t} \mathrm{~d} t
$$

as identity in $\mathrm{D}_{0, \sigma}^{-1,2}\left(B_{N}\right)$. Since $k \in \mathbb{Z}$ is arbitrary, the injectivity of the Fourier transform implies $-\partial_{t} u=g$.

From Lemma 2 and the embedding of $\mathrm{W}^{1,1}\left(\mathbb{T} ; \mathrm{D}_{0, \sigma}^{-1,2}\left(B_{N}\right)\right)$ in $C\left(\mathbb{T} ; \mathrm{D}_{0, \sigma}^{-1,2}\left(B_{N}\right)\right)$, we have, at this point, already established a continuity property of the solution from Theorem 2. We can, however, improve on this property. For this purpose, we need the following lemma:

Lemma 3. Assume that $u \in \mathrm{L}^{\infty}\left(\mathbb{T} ; \mathrm{L}_{\sigma}^{2}\left(\mathbb{R}^{3}\right)\right)$ satisfies

$$
\forall N \in \mathbb{N}: \quad u \in \mathrm{W}^{1,1}\left(\mathbb{T} ; \mathrm{D}_{0, \sigma}^{-1,2}\left(B_{N}\right)\right)
$$

Then there is a subset $\mathcal{N} \subset \mathbb{T}$ of measure zero such that

$$
\forall \varphi \in \mathrm{D}_{0, \sigma}^{1,2}\left(\mathbb{R}^{3}\right) \forall s, t \in \mathbb{T} \backslash \mathcal{N}: \quad\langle u(t), \varphi\rangle-\langle u(s), \varphi\rangle=\int_{s}^{t}\left\langle\partial_{t} u(\tau), \varphi\right\rangle \mathrm{d} \tau
$$

and $u \in C\left(\mathbb{T} \backslash \mathcal{N} ; \mathrm{L}_{w}^{2}\left(\mathbb{R}^{3}\right)^{3}\right)$, where $\mathrm{L}_{w}^{2}\left(\mathbb{R}^{3}\right)^{3}$ denotes the space $\mathrm{L}^{2}\left(\mathbb{R}^{3}\right)^{3}$ endowed with the weak topology.

Proof. It is well-known that for any function $u \in \mathrm{W}^{1,1}\left(\mathbb{T} ; \mathrm{D}_{0, \sigma}^{-1,2}\left(B_{N}\right)\right)$ there is a subset $\mathcal{N}_{N} \subset \mathbb{T}$ of measure zero such that

$$
\forall s, t \in \mathbb{T} \backslash \mathcal{N}_{N}: \quad u(t)-u(s)=\int_{s}^{t} \partial_{t} u(\tau) \mathrm{d} \tau
$$

as identity in $\mathrm{D}_{0, \sigma}^{-1,2}\left(B_{N}\right)$. It follows that $u \in C\left(\mathbb{T} \backslash \mathcal{N}_{N} ; \mathrm{D}_{0, \sigma}^{-1,2}\left(B_{N}\right)\right)$. We now put $\mathcal{N}:=$ $\bigcup_{N \in \mathbb{N}} \mathcal{N}_{N}$. It is easy to deduce that (69) is a consequence of (70). We verify that $u \in$ $C\left(\mathbb{T} \backslash \mathcal{N} ; \mathrm{L}_{w}^{2}\left(\mathbb{R}^{3}\right)^{3}\right)$. For this purpose, let $h \in \mathrm{L}^{2}\left(\mathbb{R}^{3}\right)^{3}$ and consider $u(t)$, which by assumption lies in $\mathrm{L}_{\sigma}^{2}\left(\mathbb{R}^{3}\right)$, as a linear functional on $\mathrm{L}^{2}\left(\mathbb{R}^{3}\right)^{3}$. According to the Helmholtz-Weyl decomposition (see for example [12], Theorem III.1.2), we can write $h$ as $h=H+\nabla p$ with $H \in \mathrm{L}_{\sigma}^{2}\left(\mathbb{R}^{3}\right)$ and $p \in \mathrm{W}_{\text {loc }}^{1,2}\left(\mathbb{R}^{3}\right)$. Let $\left\{\varphi_{n}\right\}_{n=1}^{\infty} \subset C_{0, \sigma}^{\infty}\left(\mathbb{R}^{3}\right)$ with $\lim _{n \rightarrow \infty} \varphi_{n}=H$ in $\mathrm{L}^{2}\left(\mathbb{R}^{3}\right)^{3}$. For any $t \in \mathbb{T} \backslash \mathcal{N}$ and sequence $\left\{t_{m}\right\}_{m=1}^{\infty} \subset \mathbb{T} \backslash \mathcal{N}^{\infty}$ with $\lim _{m \rightarrow \infty} t_{m}=t$, we deduce

$$
\begin{aligned}
\left|\left\langle u\left(t_{m}\right), h\right\rangle-\langle u(t), h\rangle\right| & =\left|\left\langle u\left(t_{m}\right), H\right\rangle-\langle u(t), H\rangle\right| \\
& \leq\left|\left\langle u\left(t_{m}\right), H-\varphi_{n}\right\rangle\right|+\left|\left\langle u\left(t_{m}\right)-u(t), \varphi_{n}\right\rangle\right|+\left|\left\langle u(t), \varphi_{n}-H\right\rangle\right| \\
& \leq 2\|u\|_{\mathrm{L}^{\infty}\left(\mathbb{T} ; \mathrm{L}^{2}\left(\mathbb{R}^{3}\right)\right)}\left\|H-\varphi_{n}\right\|_{2}+\left\|u\left(t_{m}\right)-u(t)\right\|_{\mathrm{D}_{0, \sigma}^{-1,2}\left(B_{N}\right)}\left|\varphi_{n}\right|_{1,2}
\end{aligned}
$$

provided $N$ is so large that supp $\varphi_{n} \subset B_{N}$. For arbitrary $\varepsilon>0$, we can now choose $n$ sufficiently large and, subsequently, utilize that $u \in C\left(\mathbb{T} \backslash \mathcal{N}_{N} ; \mathrm{D}_{0, \sigma}^{-1,2}\left(B_{N}\right)\right)$ to deduce

$$
\left|\left\langle u\left(t_{m}\right), h\right\rangle-\langle u(t), h\rangle\right|<\varepsilon
$$

for sufficiently large $m$.

Finally, we can show that the solution $u$ from Theorem 2 can in fact be modified on the null set $\mathcal{N}$ in such a way that $u$ becomes continuous on the whole torus. Since $u=\mathcal{P} u+\mathcal{P}_{\perp} u$ and $\mathcal{P} u$ is time independent, if suffices to establish continuity only for $\mathcal{P}_{\perp} u$. 
Theorem 3. Let $f \in \mathrm{L}^{2}\left(\mathbb{T} ; \mathrm{D}_{0}^{-1,2}\left(\mathbb{R}^{3}\right)^{3}\right)$ and $\lambda \in \mathbb{R}$. The weak time-periodic solution $u \in$ $\mathrm{L}^{2}\left(\mathbb{T} ; \mathrm{D}_{0, \sigma}^{1,2}\left(\mathbb{R}^{3}\right)\right)$ from Theorem 2 can be modified on a set of measure zero such that

$$
\mathcal{P}_{\perp} u \in C\left(\mathbb{T} ; \mathrm{L}_{w}^{2}\left(\mathbb{R}^{3}\right)^{3}\right),
$$

where again $\mathrm{L}_{w}^{2}\left(\mathbb{R}^{3}\right)^{3}$ denotes the space $\mathrm{L}^{2}\left(\mathbb{R}^{3}\right)^{3}$ endowed with the weak topology.

Proof. Theorem 2 yields $\mathcal{P}_{\perp} u \in \mathrm{L}^{\infty}\left(\mathbb{T} ; \mathrm{L}_{\sigma}^{2}\left(\mathbb{R}^{3}\right)\right)$. Thus, after possibly modifying $\mathcal{P}_{\perp} u$ on a set of measure zero, we may assume that $K:=\left\{\mathcal{P}_{\perp} u(t) \mid t \in \mathbb{T}\right\}$ is a bounded subset of $\mathrm{L}_{\sigma}^{2}\left(\mathbb{R}^{3}\right)$. Consequently, by Alaoglu's theorem, $K$ is a compact subset of $\mathrm{L}_{w}^{2}\left(\mathbb{R}^{3}\right)^{3}$. By Lemma 2, $\mathcal{P}_{\perp} u$ satisfies (68) and we can, thus, employ Lemma 3. We let $\mathcal{N}$ denote the null set from Lemma 3 and consider some $t \in \mathcal{N}$. Since $\mathcal{N}$ is a null set, there is a sequence $\left\{t_{n}\right\}_{n=1}^{\infty} \subset \mathbb{T} \backslash \mathcal{N}$ with $\lim _{n \rightarrow \infty} t_{n}=t$. The compactness of $K$ implies the existence of an element $u_{\perp}^{t} \in \mathrm{L}_{\sigma}^{2}\left(\mathbb{R}^{3}\right)$ and a subsequence of $\left\{t_{n}\right\}_{n=1}^{\infty}$, for simplicity still denoted $\left\{t_{n}\right\}_{n=1}^{\infty}$, such that $\lim _{n \rightarrow \infty} \mathcal{P}_{\perp} u\left(t_{n}\right)=u_{\perp}^{t}$ in $\mathrm{L}_{w}^{2}\left(\mathbb{R}^{3}\right)^{3}$. Let $\left\{s_{m}\right\}_{m=1}^{\infty} \subset \mathbb{T} \backslash \mathcal{N}$ be another sequence with $\lim _{m \rightarrow \infty} s_{m}=t$. We shall verify that also $\lim _{m \rightarrow \infty} \mathcal{P}_{\perp} u\left(s_{m}\right)=u_{\perp}^{t}$ in $\mathrm{L}_{w}^{2}\left(\mathbb{R}^{3}\right)^{3}$. Consider, for this purpose, an arbitrary $h \in \mathrm{L}^{2}\left(\mathbb{R}^{3}\right)^{3}$, which by the Helmholtz-Weyl decomposition can be decomposed as $h=H+\nabla p$ with $H \in \mathrm{L}_{\sigma}^{2}\left(\mathbb{R}^{3}\right)$ and $p \in \mathrm{W}_{\text {loc }}^{1,2}\left(\mathbb{R}^{3}\right)$. Let $\left\{\varphi_{k}\right\}_{k=1}^{\infty} \subset C_{0, \sigma}^{\infty}\left(\mathbb{R}^{3}\right)$ with $\lim _{k \rightarrow \infty} \varphi_{k}=H$ in $\mathrm{L}^{2}\left(\mathbb{R}^{3}\right)^{3}$. We have

$$
\begin{aligned}
\left\langle\mathcal{P}_{\perp} u\left(s_{m}\right)-u_{\perp}^{t}, h\right\rangle= & \left\langle\mathcal{P}_{\perp} u\left(s_{m}\right)-u_{\perp}^{t}, H\right\rangle \\
= & \left\langle\mathcal{P}_{\perp} u\left(s_{m}\right)-u_{\perp}^{t}, H-\varphi_{k}\right\rangle \\
& +\left\langle\mathcal{P}_{\perp} u\left(s_{m}\right)-\mathcal{P}_{\perp} u\left(t_{n}\right), \varphi_{k}\right\rangle \\
& +\left\langle\mathcal{P}_{\perp} u\left(t_{n}\right)-u_{\perp}^{t}, \varphi_{k}\right\rangle .
\end{aligned}
$$

Let $\varepsilon>0$. Recalling (69) to estimate the second term on the right-hand side in (72), we choose first $k$ and then $n$ so large that

$$
\left|\left\langle\mathcal{P}_{\perp} u\left(s_{m}\right)-u_{\perp}^{t}, h\right\rangle\right|<\varepsilon
$$

for sufficiently large $m$. We conclude that $\lim _{m \rightarrow \infty} \mathcal{P}_{\perp} u\left(s_{m}\right)=u_{\perp}^{t}$ in $\mathrm{L}_{w}^{2}\left(\mathbb{R}^{3}\right)^{3}$. We have

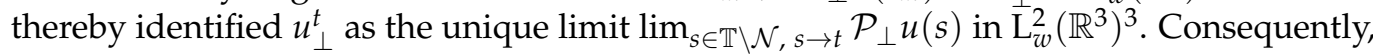

$$
u_{\perp}(t):= \begin{cases}\mathcal{P}_{\perp} u(t) & \text { if } t \in \mathbb{T} \backslash \mathcal{N}, \\ u_{\perp}^{t} & \text { if } t \in \mathcal{N}\end{cases}
$$

is well-defined. We verify that $u_{\perp} \in C\left(\mathbb{T} ; \mathrm{L}_{w}^{2}\left(\mathbb{R}^{3}\right)^{3}\right)$. Let $t \in \mathbb{T}, h \in \mathrm{L}^{2}\left(\mathbb{R}^{3}\right)^{3}$, and $\left\{t_{n}\right\}_{n=1}^{\infty} \subset$ $\mathbb{T}$ with $\lim _{n \rightarrow \infty} t_{n}=t$. If $t_{n} \in \mathbb{T} \backslash \mathcal{N}$, put $\tilde{t}_{n}:=t_{n}$. If $t_{n} \in \mathcal{N}$, we choose $\tilde{t}_{n} \in \mathbb{T} \backslash \mathcal{N}$ such that $\left|\left\langle u_{\perp}\left(t_{n}\right)-u_{\perp}\left(\tilde{t}_{n}\right), h\right\rangle\right|<\frac{1}{n}$. Then,

$$
\left|\left\langle u_{\perp}\left(t_{n}\right)-u_{\perp}(t), h\right\rangle\right| \leq\left|\left\langle u_{\perp}\left(t_{n}\right)-u_{\perp}\left(\tilde{t}_{n}\right), h\right\rangle\right|+\left|\left\langle u_{\perp}\left(\tilde{t}_{n}\right)-u_{\perp}(t), h\right\rangle\right| \rightarrow 0 \text { as } n \rightarrow \infty \text {. }
$$

Hence, $u_{\perp} \in C\left(\mathbb{T} ; \mathrm{L}_{w}^{2}\left(\mathbb{R}^{3}\right)^{3}\right)$. Since $u_{\perp}$ is a modification of $\mathcal{P}_{\perp} u$ on a null set, we conclude the lemma.

Remark 6. It was originally shown by Hopf in [1] that the weak solution to the Navier-Stokes initial-value problem established by Leray in [2] belongs to the space $C\left([0, T) ; \mathrm{L}_{w}^{2}\left(\mathbb{R}^{3}\right)^{3}\right)$. The proof of Theorem 3 essentially follows the proof in [1]; see also [17], Lemma 2.2.

\section{Existence of a Pressure}

We end by constructing a pressure term $\mathfrak{p}$ associated to the weak solution from Theorem 2. The existence of such a pressure term implies that the weak solution is, in fact, a solution in a standard sense of distributions. It is easy to verify that a completely analogous statement follows for the solution in Theorem 1. 
Two methods for constructing the pressure are given. The first method, presented in Theorem 4 below, is very general. It requires no more regularity on the data than was needed in Theorem 2 to show the existence of a weak solution. Moreover, it does not exploit that (1) is a whole-space problem and can, thus, be easily adapted to more general domains. It does, however, only produce a pressure with very little regularity in the time variable.

Theorem 4. Let $f \in \mathrm{L}^{2}\left(\mathbb{T} ; \mathrm{D}_{0}^{-1,2}\left(\mathbb{R}^{3}\right)^{3}\right)$ and $\lambda \in \mathbb{R}$. Let $u \in \mathrm{L}^{2}\left(\mathbb{T} ; \mathrm{D}_{0, \sigma}^{1,2}\left(\mathbb{R}^{3}\right)\right)$ be a weak solution to (1) that satisfies

$$
\begin{aligned}
& \mathcal{P} u \in \mathrm{D}_{0, \sigma}^{1,2}\left(\mathbb{R}^{3}\right), \\
& \mathcal{P}_{\perp} u \in \mathrm{L}^{2}\left(\mathbb{T} ; \mathrm{D}_{0, \sigma}^{1,2}\left(\mathbb{R}^{3}\right)\right) \cap \mathrm{L}^{\infty}\left(\mathbb{T} ; \mathrm{L}_{\sigma}^{2}\left(\mathbb{R}^{3}\right)\right) .
\end{aligned}
$$

Then there is a

$$
\mathfrak{p} \in \mathcal{D}^{\prime}\left(\mathbb{T} \times \mathbb{R}^{3}\right)
$$

(see Remark 7 for the definition of the space $\mathcal{D}^{\prime}\left(\mathbb{T} \times \mathbb{R}^{3}\right)$ ) such that

$$
\begin{aligned}
& \forall \Phi \in C_{0}^{\infty}\left(\mathbb{T} \times \mathbb{R}^{3}\right)^{3}: \\
& \quad \int_{\mathbb{T}} \int_{\mathbb{R}^{3}}-u \cdot \partial_{t} \Phi+\nabla u: \nabla \Phi-\lambda \partial_{1} u \cdot \Phi+(u \cdot \nabla u) \cdot \Phi \mathrm{d} x \mathrm{~d} t=\int_{\mathbb{T}}\langle f, \Phi\rangle \mathrm{d} t-\langle\nabla \mathfrak{p}, \Phi\rangle .
\end{aligned}
$$

Remark 7. We use $\mathcal{D}^{\prime}\left(\mathbb{T} \times \mathbb{R}^{3}\right)$ to denote the dual space of $\mathcal{D}\left(\mathbb{T} \times \mathbb{R}^{3}\right)$, which is defined as the vector space $C_{0}^{\infty}\left(\mathbb{T} \times \mathbb{R}^{3}\right)$ equipped with the canonical inductive limit topology. In other words, $\mathcal{D}^{\prime}\left(\mathbb{T} \times \mathbb{R}^{3}\right)$ is the space of distributions on $\mathbb{T} \times \mathbb{R}^{3}$. For $\mathfrak{p} \in \mathcal{D}^{\prime}\left(\mathbb{T} \times \mathbb{R}^{3}\right)$, the gradient $\nabla p$ is defined in the standard distributional sense. We do not need specific properties of the space $\mathcal{D}^{\prime}\left(\mathbb{T} \times \mathbb{R}^{3}\right)$ as it is only used to characterize the pressure in $(75)$.

Proof of Theorem 4. In the proof, we utilize the Fourier transform. The function spaces in the following will, therefore, all be complexified. As a consequence, the pressure term $\mathfrak{p}$ that will be constructed may be a complex-valued distribution. It is obvious, however, that the real part of $\mathfrak{p}$ will also satisfy (76).

We proceed with the proof. Put $v:=\mathcal{P} u$ and $w:=\mathcal{P}_{\perp} u$. The fact that $u$ is a weak solution to (1) implies that $w$ satisfies

$$
\begin{aligned}
& \forall \Phi \in C_{0, \sigma}^{\infty}\left(\mathbb{T} \times \mathbb{R}^{3}\right): \\
& \quad \int_{\mathbb{T}} \int_{\mathbb{R}^{3}}-w \cdot \partial_{t} \Phi+\nabla w: \nabla \Phi-\lambda \partial_{1} w \cdot \Phi \\
& \quad+\left(\mathcal{P}_{\perp}[w \cdot \nabla w]+w \cdot \nabla v+v \cdot \nabla w\right) \cdot \Phi \mathrm{d} x \mathrm{~d} t=\int_{\mathbb{T}}\left\langle\mathcal{P}_{\perp} f, \Phi\right\rangle \mathrm{d} t
\end{aligned}
$$

and $v$ satisfies

$$
\begin{aligned}
& \forall \varphi \in C_{0, \sigma}^{\infty}\left(\mathbb{R}^{3}\right): \\
& \quad \int_{\mathbb{R}^{3}} \nabla v: \nabla \varphi-\lambda \partial_{1} v \cdot \varphi+(v \cdot \nabla v+\mathcal{P}[w \cdot \nabla w]) \cdot \varphi \mathrm{d} x \mathrm{~d} t=\langle\mathcal{P} f, \varphi\rangle .
\end{aligned}
$$


We shall construct a pressure term for the weak formulations (77) and (78) separately. We start with (77). Observe that

$$
\begin{aligned}
& \int_{\mathbb{T}}|w \cdot \nabla w|_{-1,2} \mathrm{~d} t=\int_{\mathbb{T}} \sup _{\substack{\varphi \in C_{0}^{\infty}\left(\mathbb{R}^{3}\right)^{3} \\
|\varphi|_{12} \leq 1}}\left|\int_{\mathbb{R}^{3}}(w \cdot \nabla w) \cdot \varphi \mathrm{d} x\right| \mathrm{d} t \\
& =\int_{\mathbb{T}} \sup _{\substack{\varphi \in C_{0}^{\infty}\left(\mathbb{R}^{3}\right)^{3} \\
|\varphi| 1,2 \leq 1}}\left|\int_{\mathbb{R}^{3}} w \otimes w: \nabla \varphi \mathrm{d} x\right| \mathrm{d} t \\
& \leq c_{1} \int_{\mathbb{T}}\|w(t)\|_{4}^{2} \mathrm{~d} t \\
& \leq c_{2} \int_{\mathbb{T}}\|w(t)\|_{2}^{\frac{1}{2}}\|\nabla w(t)\|_{2}^{\frac{3}{2}} \mathrm{~d} t \\
& \leq c_{3}\|w\|_{\mathrm{L}^{\infty}\left(\mathbb{T} ; \mathrm{L}^{2}\left(\mathbb{R}^{3}\right)\right)}^{\frac{1}{2}}\|w\|_{\mathrm{L}^{2}\left(\mathbb{T} ; \mathrm{D}_{0, \sigma}^{1,2}\left(\mathbb{R}^{3}\right)\right)^{\prime}}
\end{aligned}
$$

where the second-last inequality is due to Lemma 1. It follows that

$$
w \cdot \nabla w \in \mathrm{L}^{1}\left(\mathbb{T} ; \mathrm{D}_{0}^{-1,2}\left(\mathbb{R}^{3}\right)^{3}\right) .
$$

By a similar computation we find that

$$
\begin{aligned}
\int_{\mathbb{T}}|w \cdot \nabla v|_{-1,2} \mathrm{~d} t & =\int_{\substack { \mathbb{T} \\
\begin{subarray}{c}{\varphi \in C_{0}^{\infty}\left(\mathbb{R}^{3}\right)^{3} \\
|\varphi|_{1,2} \leq 1{ \mathbb { T } \\
\begin{subarray} { c } { \varphi \in C _ { 0 } ^ { \infty } ( \mathbb { R } ^ { 3 } ) ^ { 3 } \\
| \varphi | _ { 1 , 2 } \leq 1 } }\end{subarray}}\left|\int_{\mathbb{R}^{3}} v \otimes w: \nabla \varphi \mathrm{d} x\right| \mathrm{d} t \\
& \leq c_{4} \int_{\mathbb{T}}\|v\|_{6}\|w(t)\|_{3} \mathrm{~d} t \\
& \leq c_{5}\|\nabla v\|_{2} \int_{\mathbb{T}}\|w(t)\|_{2}^{\frac{1}{2}}\|\nabla w(t)\|_{2}^{\frac{1}{2}} \mathrm{~d} t \\
& \leq c_{6}|v|_{1,2}\|w\|_{L^{\infty}\left(\mathbb{T} ; \mathrm{L}^{2}\left(\mathbb{R}^{3}\right)\right)}^{\frac{1}{2}}\|w\|_{\mathrm{L}^{2}\left(\mathbb{T} ; \mathrm{D}_{0, \sigma}^{1,2}\left(\mathbb{R}^{3}\right)\right)^{\prime}}
\end{aligned}
$$

and thus

$$
w \cdot \nabla v \in \mathrm{L}^{1}\left(\mathbb{T} ; \mathrm{D}_{0}^{-1,2}\left(\mathbb{R}^{3}\right)^{3}\right)
$$

Analogously, we deduce

$$
v \cdot \nabla w \in \mathrm{L}^{1}\left(\mathbb{T} ; \mathrm{D}_{0}^{-1,2}\left(\mathbb{R}^{3}\right)^{3}\right) .
$$

We now expand $u \in \mathrm{L}^{2}\left(\mathbb{T} ; \mathrm{D}_{0, \sigma}^{1,2}\left(\mathbb{R}^{3}\right)\right)$ in a Fourier series

$$
\left\{u_{k}\right\}_{k \in \mathbb{Z}} \in \ell^{2}\left(\mathbb{Z} ; D_{0, \sigma}^{1,2}\left(\mathbb{R}^{3}\right)\right) .
$$

Observe that $v=u_{0}$. Consequently, $\left\{u_{k}\right\}_{k \neq 0}$ is the Fourier series of $w$, whence

$$
\left\{u_{k}\right\}_{k \neq 0} \in \ell^{2}\left(\mathbb{Z} ; \mathrm{L}^{2}\left(\mathbb{R}^{3}\right)^{3}\right) .
$$

We further put

$$
H:=\mathcal{P}_{\perp}[u \cdot \nabla u]=\mathcal{P}_{\perp}[w \cdot \nabla w]+w \cdot \nabla v+v \cdot \nabla w .
$$

The definition of $H$ requires that we identify $u \cdot \nabla u$ as an element of a function space on which $\mathcal{P}_{\perp}$ is well-defined. For this purpose, we may, for example, choose the space 
$\mathrm{L}^{1}\left(\mathbb{T} ; \mathrm{W}_{0}^{-1,2}\left(\mathbb{R}^{3}\right)^{3}\right)$. In view of (79)-(81), $H \in \mathrm{L}^{1}\left(\mathbb{T} ; \mathrm{D}_{0}^{-1,2}\left(\mathbb{R}^{3}\right)^{3}\right)$. We can therefore expand $H$ into a Fourier series

$$
\left\{H_{k}\right\}_{k \in \mathbb{Z}} \in \ell^{\infty}\left(\mathbb{Z} ; D_{0}^{-1,2}\left(\mathbb{R}^{3}\right)^{3}\right) .
$$

Observe that $H_{0}=\mathscr{F}_{\mathbb{T}}[H](0)=\mathcal{P} H=0$. Finally, we also expand $f$ into a Fourier series

$$
\left\{f_{k}\right\}_{k \in \mathbb{Z}} \in \ell^{2}\left(\mathbb{Z} ; \mathrm{D}_{0}^{-1,2}\left(\mathbb{R}^{3}\right)^{3}\right) .
$$

For $k \in \mathbb{Z} \backslash\{0\}$ and $N \in \mathbb{N}$, we define

$$
\begin{aligned}
& \mathcal{L}_{k}^{N}: \mathrm{W}_{0}^{1,2}\left(B_{N}\right)^{3} \rightarrow \mathbb{C}, \\
& \mathcal{L}_{k}^{N}(\varphi):=\int_{\mathbb{R}^{3}}-i k \frac{2 \pi}{\mathcal{T}} u_{-k} \cdot \varphi+\nabla u_{-k}: \nabla \varphi-\lambda \partial_{1} u_{-k} \cdot \varphi \mathrm{d} x+\left\langle H_{-k}, \varphi\right\rangle-\left\langle f_{-k}, \varphi\right\rangle .
\end{aligned}
$$

Recalling that $u_{k}=\mathscr{F}_{\mathbb{T}}[w](k)$ and, thus, $u_{k} \in \mathrm{L}^{2}\left(\mathbb{R}^{3}\right)^{3}$, it is easy to see that $\mathcal{L}_{k}^{N}$ is a bounded linear functional on $\mathrm{W}_{0}^{1,2}\left(B_{N}\right)^{3}$. Consider now an element $\varphi \in C_{0, \sigma}^{\infty}\left(\mathbb{R}^{3}\right)$. The function $\Phi(t, x):=\varphi(x) \mathrm{e}^{\frac{2 \pi}{T} k t}$ is then admissible as a test function in (77), which implies that $\mathcal{L}_{k}^{N}(\varphi)=0$. Thus, $\mathcal{L}_{k}^{N}$ vanishes on $C_{0, \sigma}^{\infty}\left(\mathbb{R}^{3}\right)$. By a well-known theorem, see for example [12] (Corollary III.5.1), which easily extends by linearity to complexified vector spaces, it follows that there is $\mathfrak{p}_{k}^{N} \in \mathrm{L}^{2}\left(B_{N}\right)$ such that $\mathcal{L}_{k}^{N}=\nabla \mathfrak{p}_{k}^{N}$; that is,

$$
\forall \varphi \in C_{0}^{\infty}\left(B_{N}\right)^{3}: \quad \mathcal{L}_{k}^{N}(\varphi)=-\int_{\mathbb{R}^{3}} \mathfrak{p}_{k}^{N} \operatorname{div} \varphi \mathrm{d} x .
$$

Clearly, $\mathfrak{p}_{k}^{N}$ can be chosen such that

$$
\int_{B_{N}} \mathfrak{p}_{k}^{N} \mathrm{~d} x=0
$$

Consequently—see for example [12] (Theorem III.3.1)—there is a $\varphi \in \mathrm{W}_{0}^{1,2}\left(B_{N}\right)^{3}$ with the properties $\operatorname{div} \varphi=-\mathfrak{p}_{k}^{N}$ and $|\varphi|_{1,2} \leq c_{7}\left\|\mathfrak{p}_{k}^{N}\right\|_{2}$. Inserting this particular $\varphi$ in (87), we find that $\left\|\mathfrak{p}_{k}^{N}\right\|_{2}^{2}=\mathcal{L}_{k}^{N}(\varphi)$. It follows that

$$
\left\|\mathfrak{p}_{k}^{N}\right\|_{2} \leq c_{8}\left(|k|\left\|u_{-k}\right\|_{2}+\left\|\nabla u_{-k}\right\|_{2}+\left|H_{-k}\right|_{-1,2}+\left|f_{-k}\right|_{-1,2}\right)
$$

with $c_{8}=c_{8}(N)$. Recalling (82)-(85), we see that

$$
\left\{\frac{1}{k} \mathfrak{p}_{k}^{N}\right\}_{k \neq 0} \in \ell^{2}\left(\mathbb{Z} ; \mathrm{L}^{2}\left(B_{N}\right)\right)
$$

We can, thus, define

$$
\mathfrak{P}^{N}:=-i \frac{\mathcal{T}}{2 \pi} \mathscr{F}_{\mathbb{T}}^{-1}\left[\left\{\frac{1}{k} \mathfrak{p}_{k}^{N}\right\}_{k \neq 0}\right] \in \mathrm{L}^{2}\left(\mathbb{T} ; \mathrm{L}^{2}\left(B_{N}\right)\right) .
$$

Consider now $N, M \in \mathbb{N}$ with $N<M$. For any $\varphi \in C_{0}^{\infty}\left(B_{N}\right)^{3}$, it follows from (87) that $\left\langle\mathfrak{P}^{N}(\cdot), \operatorname{div} \varphi\right\rangle_{\mathrm{L}^{2}\left(B_{N}\right)}=\left\langle\mathfrak{P}^{M}(\cdot), \operatorname{div} \varphi\right\rangle_{\mathrm{L}^{2}\left(B_{N}\right)}$ as an equality in $\mathrm{L}^{2}(\mathbb{T} ; \mathbb{C})$. Consequently, $\nabla \mathfrak{P}^{N}(t)=\nabla \mathfrak{P}^{M}(t)$ in $\mathrm{D}_{0}^{-1,2}\left(B_{N}\right)^{3}$ for almost all $t \in \mathbb{T}$. We, therefore, find that $\mathfrak{P}^{N}(t)=$ $\mathfrak{P}^{M}(t)+c(N, M, t)$ in $\mathrm{L}^{2}\left(B_{N}\right)$ for some function $c(N, M, \cdot) \in \mathrm{L}^{2}(\mathbb{T})$. Letting

$$
\widetilde{\mathfrak{P}}^{1}(t):=\mathfrak{P}^{1}(t), \quad \widetilde{\mathfrak{P}}^{N+1}(t):=\mathfrak{P}^{N+1}(t)+c(N, N+1, t)+\left(\widetilde{\mathfrak{P}}^{N}(t)-\mathfrak{P}^{N}(t)\right),
$$


we inductively obtain a sequence $\left\{\widetilde{\mathfrak{P}}^{N}\right\}_{N=1}^{\infty}$ with $\widetilde{\mathfrak{P}}^{M}=\widetilde{\mathfrak{P}}^{N}$ in $\mathrm{L}^{2}\left(\mathbb{T} ; \mathrm{L}^{2}\left(B_{N}\right)\right)$ for $M \geq N$. At this point, we can define an element in $\mathfrak{P} \in \mathrm{L}^{2}\left(\mathbb{T} ; \mathrm{L}_{\text {loc }}^{2}\left(\mathbb{R}^{3}\right)\right)$ via $\mathfrak{P}(t, x):=\widetilde{\mathfrak{P}}^{N}(t, x)$ if $|x| \leq N$. We can consider $\mathfrak{P}$ as an element in $\mathcal{D}^{\prime}\left(\mathbb{T} \times \mathbb{R}^{3}\right)$ and put

$$
\pi:=\partial_{t} \mathfrak{P} \in \mathcal{D}^{\prime}\left(\mathbb{T} \times \mathbb{R}^{3}\right) .
$$

We now verify that $\pi$, as defined above, is a valid pressure term corresponding to the weak formulation (77). For this purpose, we consider $\Phi \in C_{0}^{\infty}\left(\mathbb{T} \times \mathbb{R}^{3}\right)^{3}$ and observe that

$$
\begin{aligned}
\langle\nabla \pi, \Phi\rangle=\left\langle\mathfrak{P}, \partial_{t} \operatorname{div} \Phi\right\rangle & =\int_{\mathbb{T}} \int_{\mathbb{R}^{3}} \mathfrak{P}(t, x) \cdot \partial_{t} \operatorname{div} \Phi(t, x) \mathrm{d} x \mathrm{~d} t \\
& =\int_{\mathbb{T}} \int_{\mathbb{R}^{3}} \mathfrak{P}^{N}(t, x) \cdot \partial_{t} \operatorname{div} \Phi(t, x) \mathrm{d} x \mathrm{~d} t
\end{aligned}
$$

for $N \in \mathbb{N}$ with $\operatorname{supp}(\Phi) \subset B_{N} \times \mathbb{T}$. Recalling (88), it follows that

$$
\langle\nabla \pi, \Phi\rangle=-i \frac{\mathcal{T}}{2 \pi} \int_{\mathbb{T}}\left\langle\mathscr{F}_{\mathbb{T}}^{-1}\left[\left\{\frac{1}{k} \mathfrak{p}_{k}^{N}\right\}_{k \neq 0}\right], \overline{\partial_{t} \operatorname{div} \Phi(t, \cdot)}\right\rangle_{\mathrm{L}^{2}\left(B_{N}\right)} \mathrm{d} t .
$$

Recall that, in this proof, we regard $\mathrm{L}^{2}\left(B_{N}\right)$ as a vector space over $\mathbb{C}$, which is the reason the complex conjugation of $\partial_{t} \operatorname{div} \Phi(t, x)$ appears in the equation above. By Parseval's identity, we deduce that

$$
\langle\nabla \pi, \Phi\rangle=\sum_{k \in \mathbb{Z} \backslash\{0\}}-i \frac{\mathcal{T}}{2 \pi}\left\langle\frac{1}{k} \mathfrak{p}_{k}^{N},-i \frac{2 \pi}{\mathcal{T}} k \operatorname{div} \bar{\Phi}_{k}\right\rangle_{\mathrm{L}^{2}\left(B_{N}\right)^{\prime}}
$$

where $\left\{\Phi_{k}\right\}_{k \in \mathbb{Z}} \in \ell^{2}\left(\mathbb{Z} ; \mathrm{L}^{2}\left(\mathbb{R}^{3}\right)^{3}\right)$ is the Fourier transform $\mathscr{F}_{\mathbb{T}}[\Phi]$ of $\Phi$ with respect to the $t$-variable. Clearly, $\Phi_{k}$ enjoys the same regularity as $\Phi$ with respect to the $x$-variable. In other words, $\Phi_{k} \in C_{0}^{\infty}\left(\mathbb{R}^{3}\right)^{3}$. Recalling (87), we, therefore, see that

$$
\begin{aligned}
\langle\nabla \pi, \Phi\rangle= & \sum_{k \in \mathbb{Z} \backslash\{0\}}\left\langle\mathfrak{p}_{k}^{N},-\operatorname{div} \bar{\Phi}_{k}\right\rangle_{\mathrm{L}^{2}\left(B_{N}\right)} \\
= & \sum_{k \in \mathbb{Z} \backslash\{0\}} \mathcal{L}_{k}^{N}\left(\Phi_{k}\right) \\
= & \sum_{k \in \mathbb{Z} \backslash\{0\}} \int_{\mathbb{R}^{3}}-i k \frac{2 \pi}{\mathcal{T}} u_{-k} \cdot \Phi_{k}+\nabla u_{-k}: \nabla \Phi_{k}-\lambda \partial_{1} u_{-k} \cdot \Phi_{k} \mathrm{~d} x \\
& \quad+\left\langle H_{-k}, \Phi_{k}\right\rangle-\left\langle f_{-k}, \Phi_{k}\right\rangle .
\end{aligned}
$$

A Parseval-type identity holds for all terms on the right-hand side above. For example, utilizing Fubini's theorem and the fact that the Fourier series $\sum_{k \in \mathbb{Z} \backslash\{0\}} \Phi_{k} \mathrm{e}^{i \frac{2 \pi}{T} k t}$ converges pointwise for each $t \in \mathbb{T}$ towards $\mathcal{P}_{\perp} \Phi$ in the topology of $\mathrm{D}_{0}^{1,2}\left(\mathbb{R}^{3}\right)$, we obtain

$$
\begin{aligned}
\sum_{k \in \mathbb{Z} \backslash\{0\}}\left\langle H_{-k}, \Phi_{k}\right\rangle & =\sum_{k \in \mathbb{Z} \backslash\{0\}}\left\langle\int_{\mathbb{T}} H(t) \mathrm{e}^{i \frac{2 \pi}{T} k t} \mathrm{~d} t, \Phi_{k}\right\rangle \\
& =\int_{\mathbb{T}}\left\langle H(t), \sum_{k \in \mathbb{Z} \backslash\{0\}} \Phi_{k} \mathrm{e}^{i \frac{2 \pi}{T} k t}\right\rangle \mathrm{d} t=\int_{\mathbb{T}}\left\langle H(t), \mathcal{P}_{\perp} \Phi\right\rangle \mathrm{d} t .
\end{aligned}
$$

Recalling that $\mathcal{P} H=0$, the above identity implies

$$
\begin{aligned}
\sum_{k \in \mathbb{Z} \backslash\{0\}}\left\langle H_{-k}, \Phi_{k}\right\rangle & =\int_{\mathbb{T}}\langle H(t), \Phi\rangle \mathrm{d} t \\
& =\int_{\mathbb{T}} \int_{\mathbb{R}^{3}}\left(\mathcal{P}_{\perp}[w \cdot \nabla w]+w \cdot \nabla v+v \cdot \nabla w\right) \cdot \Phi(t, x) \mathrm{d} x \mathrm{~d} t
\end{aligned}
$$


Applying a similar computation in the other terms on the right-hand side in (90), we obtain

$$
\begin{aligned}
\langle\nabla \pi, \Phi\rangle=\int_{\mathbb{T}} \int_{\mathbb{R}^{3}}-w \cdot \partial_{t} \Phi+\nabla w: \nabla \Phi-\lambda \partial_{1} w \cdot \Phi \\
+\left(\mathcal{P}_{\perp}[w \cdot \nabla w]+w \cdot \nabla v+v \cdot \nabla w\right) \cdot \Phi \mathrm{d} x \mathrm{~d} t-\int_{\mathbb{T}}\left\langle\mathcal{P}_{\perp} f, \Phi\right\rangle \mathrm{d} t .
\end{aligned}
$$

We have, thus, constructed in $\pi$ a pressure term for the weak formulation (77). The existence of a pressure term for the weak formulation (78) follows a by standard argument. More specifically, we define on the real-valued vector space $C_{0}^{\infty}\left(\mathbb{R}^{3}\right)^{3}$ the linear functional

$$
\begin{aligned}
& \mathcal{L}: C_{0}^{\infty}\left(\mathbb{R}^{3}\right)^{3} \rightarrow \mathbb{R}, \\
& \mathcal{L}(\varphi):=\int_{\mathbb{R}^{3}} \nabla v: \nabla \varphi-\lambda \partial_{1} v \cdot \varphi+(v \cdot \nabla v+\mathcal{P}[w \cdot \nabla w]) \cdot \varphi \mathrm{d} x \mathrm{~d} t-\langle\mathcal{P} f, \varphi\rangle .
\end{aligned}
$$

In view of (73) and (79), it is standard to show for each bounded domain $\Omega \subset \mathbb{R}^{3}$ that the restriction $\mathcal{L}_{\mid C_{0}^{\infty}(\Omega)^{3}}$ extends to a bounded linear functional on $\mathrm{D}_{0}^{1,2}(\Omega)^{3}$. Consequentlysee for example [12] (Corollary III.5.1)—there is $p \in \mathrm{L}_{\text {loc }}^{2}\left(\mathbb{R}^{3}\right)$ such that $\mathcal{L}=\nabla p$ in the sense of distributions. We can trivially extend $p$ to a time-independent element of $\mathrm{L}_{\text {loc }}^{2}\left(\mathbb{T} \times \mathbb{R}^{3}\right)$. Then $\nabla p \in \mathcal{D}^{\prime}\left(\mathbb{T} \times \mathbb{R}^{3}\right)^{3}$, and for each $\Phi \in C_{0}^{\infty}\left(\mathbb{T} \times \mathbb{R}^{3}\right)^{3}$ we have $\langle\nabla p, \mathcal{P} \perp \Phi\rangle=0$. Thus,

$$
\begin{aligned}
& \langle\nabla p, \Phi\rangle=\langle\nabla p, \mathcal{P} \Phi\rangle \\
& \quad=\int_{\mathbb{R}^{3}} \nabla v: \nabla \mathcal{P} \Phi-\lambda \partial_{1} v \cdot \mathcal{P} \Phi+(v \cdot \nabla v+\mathcal{P}[w \cdot \nabla w]) \cdot \mathcal{P} \Phi \mathrm{d} x \mathrm{~d} t-\langle\mathcal{P} f, \mathcal{P} \Phi\rangle
\end{aligned}
$$

We finally put

$$
\mathfrak{p}:=\pi+p
$$

and observe for arbitrary $\Phi \in C_{0}^{\infty}\left(\mathbb{T} \times \mathbb{R}^{3}\right)^{3}$, by recalling $u=v+w$ and (91)-(92), that

$$
\begin{aligned}
\int_{\mathbb{T}} \int_{\mathbb{R}^{3}}-u \cdot \partial_{t} \Phi+\nabla u: \nabla \Phi-\lambda \partial_{1} u \cdot \Phi+(u \cdot \nabla u) \cdot \Phi \mathrm{d} x \mathrm{~d} t-\int_{\mathbb{T}}\langle f, \Phi\rangle \mathrm{d} t \\
=\int_{\mathbb{T}} \int_{\mathbb{R}^{3}}-(v+w) \cdot \partial_{t} \Phi+(\nabla v+\nabla w): \nabla \Phi-\lambda\left(\partial_{1} v+\partial_{1} w\right) \cdot \Phi \\
\quad+((v+w) \cdot \nabla(v+w)) \cdot \Phi \mathrm{d} x \mathrm{~d} t \\
\quad-\int_{\mathbb{T}}\left\langle\mathcal{P}_{\perp} f, \Phi\right\rangle \mathrm{d} t-\int_{\mathbb{T}}\langle\mathcal{P} f, \Phi\rangle \mathrm{d} t \\
=\int_{\mathbb{T}} \int_{\mathbb{R}^{3}}-w \cdot \partial_{t} \Phi+\nabla w: \nabla \Phi-\lambda \partial_{1} w \cdot \Phi \\
\quad+\left(\mathcal{P}_{\perp}[w \cdot \nabla w]+w \cdot \nabla v+v \cdot \nabla w\right) \cdot \Phi \mathrm{d} x \mathrm{~d} t-\int_{\mathbb{T}}\left\langle\mathcal{P}_{\perp} f, \Phi\right\rangle \mathrm{d} t \\
\quad+\int_{\mathbb{R}^{3}} \nabla v: \nabla \mathcal{P} \Phi-\lambda \partial_{1} v \cdot \mathcal{P} \Phi+(v \cdot \nabla v+\mathcal{P}[w \cdot \nabla w]) \cdot \mathcal{P} \Phi \mathrm{d} x \mathrm{~d} t-\langle\mathcal{P} f, \mathcal{P} \Phi\rangle \\
=\langle\nabla \pi, \Phi\rangle+\langle\nabla p, \Phi\rangle \\
=\langle\nabla \mathfrak{p}, \Phi\rangle .
\end{aligned}
$$

We have, thus, established (76) and, thereby, the theorem.

Funding: This research received no external funding.

Institutional Review Board Statement: Not applicable.

Informed Consent Statement: Not applicable.

Data Availability Statement: Not applicable. 
Conflicts of Interest: The author declares no conflict of interest.

\section{References}

1. Hopf, E. Über die Anfangswertaufabe für die hydrodynamischen Grundgleichungen. Math. Nachr. 1951, 4, 213-231. [CrossRef]

2. Leray, J. Sur le mouvement d'un liquide visqueux emplissant l'espace. Acta Math. 1934, 63, 193-248. [CrossRef]

3. Galdi, G.P. An Introduction to the Mathematical Theory of the Navier-Stokes Equations. Steady-State Problems, 2nd ed.; Springer: New York, NY, USA, 2011.

4. Maremonti, P. Existence and stability of time-periodic solutions to the Navier-Stokes equations in the whole space. Nonlinearity 1991, 4, 503-529. [CrossRef]

5. Maremonti, P. Some theorems of existence for solutions of the Navier-Stokes equations with slip boundary conditions in half-space. Ric. Mat. 1991, 40, 81-135.

6. Maremonti, P.; Padula, M. Existence, uniqueness and attainability of periodic solutions of the Navier-Stokes equations in exterior domains. Zap. Nauchn. Sem. POMI 1996, 233, 142-182. [CrossRef]

7. Kozono, H.; Nakao, M. Periodic solutions of the Navier-Stokes equations in unbounded domains. Tohoku Math. J. 1996, 48, 33-50. [CrossRef]

8. Yamazaki, M. The Navier-Stokes equations in the weak- $L^{n}$ space with time-dependent external force. Math. Ann. 2000, 317, 635-675. [CrossRef]

9. Galdi, G.P.; Sohr, H. Existence and uniqueness of time-periodic physically reasonable Navier-Stokes flow past a body. Arch. Ration. Mech. Anal. 2004, 172, 363-406. [CrossRef]

10. Kyed, M. Time-Periodic Solutions to the Navier-Stokes Equations; Habilitationsschrift; Technische Universität Darmstadt: Darmstadt, Germany, 2012.

11. Galdi, G.P.; Kyed, M. Time-periodic solutions to the navier-stokes equations. In Handbook of Mathematical Analysis in Mechanics of Viscous Fluids; Giga, Y., Novotny, A., Eds.; Springer International Publishing: Cham, Switzerland, 2016; pp. 1-70.

12. Galdi, G.P. An Introduction to the Mathematical Theory of the Navier-Stokes Equations. Volume I: Linearized Steady Problems; Springer Tracts in Natural Philosophy. 38; Springer: New York, NY, USA, 1994.

13. Galdi, G.P. An Introduction to the Mathematical Theory of the Navier-Stokes Equations. Volume II: Nonlinear Steady Problems; Springer Tracts in Natural Philosophy. 39; Springer: New York, NY, USA, 1994.

14. Temam, R. Navier-Stokes Equations. Theory and Numerical Analysis; North-Holland Publishing Company: Amsterdam, The Netherlands; New York, NY, USA; Oxford, UK, 1979.

15. Finn, R. An energy theorem for viscous fluid motions. Arch. Ration. Mech. Anal. 1960, 6, 371-381. [CrossRef]

16. Korolev, A.; Šverák, V. On the large-distance asymptotics of steady state solutions of the Navier-Stokes equations in 3D exterior domains. Ann. Inst. Henri Poincaré Anal. Non Linéaire 2011, 28, 303-313. [CrossRef]

17. Galdi, G.P. An introduction to the Navier-Stokes initial-boundary value problem. In Fundamental Directions in Mathematical Fluid Mechanics; Advances in Mathematical Fluid Mechanics; Birkhäuser: Basel, Switzerland, 2000; pp. 1-70. 